Plant Production Science

http:/www.journals.zu.edu.eg/journalDisplay.aspx?Journalld=1\&queryType=Master

\title{
EFFECT OF SOME NATURAL EXTRACTS, INDOLBUTIRIC ACID AND NAPHTHALENE ACETIC ACID ON ROOTING OF PICUAL OLIVE CUTTINGS
}

\author{
Abd-Allah M.H. Eid", Safaa A. Nomier, M.M. Ibrahim and M.M. Gad \\ Hort. Dept., Fac. Agric., Zagazig Univ., Egypt
}

Received: 12/11/2017 ; Accepted: 19/12/2017

\begin{abstract}
This study was carried out during 2014 and 2015 seasons in a private plastic green house at Belbies District, Sharkia Governorate, Egypt in an attempt to replace the traditional olive cutting rooting synthetic growth regulators by some auxin releasing natural extracts. Cuttings of Picual olive cv. were prepared on November of each season (about $15 \mathrm{~cm}$ long and7-9 $\mathrm{mm}$ in diameter), cutting were dipped in concentrations of indole-3-Butyric acid (IBA) at $4000 \mathrm{ppm}$ and naphthalene acetic acid (NAA) at $500 \mathrm{ppm}$ solution for 5 seconds or soaked in the natural extracts for half hour. The results revealed that the maximum rooting percentage (81.44 and $85.60 \%)$, number of roots/cutting (14.84 and 14.90 root/cutting), shoot number/ transplant (2.74 and 2.17 shoot/plant), leaf number/plant (6.49 and 6.61 leaf/plant) and bud sprouting percentage (48.46 and $48.14 \%$ ) respectively were recorded for cuttings dipped in IBA at $4000 \mathrm{ppm}$ in comparison with those dipped in moringa extracts, which gained the lowest values of the considered parameters during the two seasons. The highest cutting survival percentages were recorded for IBA and NAA treatments, garlic at 10 or $20 \%$, liquorice at $10 \mathrm{~g}$ and algae at 2.5 or $5 \mathrm{~cm}$ in both seasons without significant differences between them. Total carbohydrates percentages in the roots and shoots were gradually increased with the advance in planting months, and reached the highest values after seven months, but total soluble phenols in the roots were decreased after six months then increased in the seventh month in both seasons. The algae, garlic and liquorice treatments were considered the best natural extracts compared to yeast and in turn can be used as an alternative of growth regulators for rooting Picual olive cuttings.
\end{abstract}

Key words: Olive, picual, rooting (\%), sprouting (\%), growth regulators, natural extracts.

\section{INTRODUCTION}

Vegetative propagation through rooting leafy cuttings in a mist system is widely used for the propagation of olive (Olea europaea L.) trees. Olives are commonly propagated by leafy cuttings under mist irrigation (Peixe et al., 2007). Stem cuttings are the most important simple and economical means of vegetative propagation. This technique is considered to be easy, inexpensive and appropriate for mass plant production within a short time, but there are great differences in the rooting potential among olive cultivars. Thus, it has been categorized into three groups, easy, moderate and hard-toroot cultivars (Fernandes et al., 2002; Pio et

\footnotetext{
* Corresponding author: Tel. : +21227022572

E-mail address: faridmsamy@yahoo.com
}

al., 2005). Although self-rooted cultivars can be very interesting in establishing new olive orchard, the low rooting ability, the unsatisfactory viability and the low rooting quality of cuttings in some cultivars represent limiting factors (Wiesman and Lavee, 1994). Between several factors, internal concentrations of auxins and carbohydrates have been reported to play an important role in rooting process. The external application of auxins is one of the most important factors in increasing the rooting ability of many species (Hartmann et al., 2002; Kelen and Ozkan, 2003; Negash, 2003). It is well known that auxins stimulate rooting of cuttings, while cytokinins and gibberellins generally inhibit rooting (Hartmann et al., 
2002). The external application of auxins, especially IBA usually increases the rooting ability of cuttings. However, IBA treatment has not always stimulate root initiation in olive (Aslmoshtaghi and Shahsavar, 2010a).

The use of auxin, mainly Indole butyric acid (IBA), has become a vital practice for induction of adventitious roots in nurseries. Various attempts have been made to enhance rooting ability of olive cultivars with wounding (Ayoub, 1995). Severance from the stock plant induces the biosynthesis of phenolic compounds, which is why many authors have detected an increased phenolic content level in the first days after the establishment of cuttings (Quaddoury and Amssa, 2004; Osterc and Štampar, 2008). After application of exogenous IBA on cuttings, the endogenous auxin concentration reaches a peak after wounding coinciding with the initation of the rooting process. Interaction between endogenous IAA and exogenous IBA during adventitious root formation has been suggested and the performance of IBA versus IAA explained by several possibilities: higher stability, differences in metabolism, differences in transport, and IBA as a slow release source of IAA. Furthermore, IBA is reported to increase the rate of ethylene biosynthesis, and the auxinethylene relationship during root development has been shown by a number of isolated mutants that have resistance to both hormones. In addition, jasmonates and cytokine are involved in the regulation of sink-source relationsships, leaf senescence, and control of meristematic activity during adventitious root formation (Perilli et al., 2010; Pop et al., 2011).

Algae extract as a new biofertilizer containing some macro (N, P, K, Ca, Mg and $\mathrm{S}$ ) and micro nutrients $(\mathrm{Zn}, \mathrm{Fe}, \mathrm{Mn}, \mathrm{Cu}, \mathrm{Mo}$ and $\mathrm{Co})$ as well as some growth regulators, polyamines and vitamins required to be applied for improve nutritional status, vegetative growth, yield and fruit quality in different fruit orchards (Abd ElMigeed et al., 2004; Abd El-Moniem and Abd-Allah, 2008; Spinelli et al., 2009). Leaves of moringa are rich in zeatin, it can be used as natural source of cytokinin (Fuglie, 2000). In addition, moringa leaves also rich in ascorbates, carotenoids, phenols, potassium and calcium, which have plant growth promoting capabilities and often applied as exogenous plant growth enhancers (Foidl et al., 2001). It also contains indole acetic acid and plant growth regulators (Sachan et al., 2011). Garlic also contains vitamins, minerals, flavonoids, ascorbic acid, sulphur and trace of iodine. Seventeen amino acids are found in garlic, including eight essential ones. Effect of garlic extract on plant characters has been interpreted by El-Shayeb (2009). The licorice extract contain more than 100 various compounds, some of which accumulated in large amounts, which most important of them are triterpene saponins (including glycyrrhizin) and phenolic compounds (Shabani et al., 2009). Agrobacterium rhizogenes induces formation of adventitious roots at sites of infection, resulting in a hairy-root phenotype. This extensive root formation is caused by expression of $A$. rhizogenes encoded by T-DNA, a fragment of DNA originating from a rootinducing plasmid (Moore et al., 1979). In addition, yeast is a natural source of many growth substances as a protective agent, and most of nutritional elements $(\mathrm{Na}, \mathrm{Ca}, \mathrm{Fe}, \mathrm{K}, \mathrm{P}, \mathrm{S}, \mathrm{Mg}$, $\mathrm{Zn}$ and $\mathrm{Si}$ ) and contains cytokinin as well as some organic compounds (Nagodawithana, 1991).

The objective of this work is finding out auxin releasing natural extracts used as cuttings rooting enhancement instead of the conventional olive rooting hormones.

\section{MATERIALS AND METHODS}

This study was carried out during two seasons of 2014 and 2015 in a private plastic greenhouse at Belbies District, Sharkia Governorate, Egypt. One-year-old shoots of the current season were collected from Picual olive cv. (Olea europea L.). The semi-hardwood cuttings (middle part portion) were prepared on November of each season of about $15 \mathrm{~cm}$ in long and 7- $9 \mathrm{~mm}$ in diameter with five nodes and two pair of leaves.

\section{The Experimental Procedures}

The prepared cuttings were subjected to the following 13 dipping and soaking treatments:

\section{Control (dipping cuttings in IBA solution at $4000 \mathrm{ppm})$}

2. Dipping cuttings in NAA solution at $500 \mathrm{ppm}$

3. Dipping cuttings in IBA solution at $4000 \mathrm{ppm}$ + NAA at $500 \mathrm{ppm}$ 
4. Soaking cuttings in moringa extract at $10 \%$.

5. Soaking cuttings in moringa extract at $20 \%$.

6. Soaking cuttings in garlic extract at $10 \%$.

7. Soaking cuttings in garlic extract at $20 \%$.

8. Soaking cuttings in liquorice extract at $10 \mathrm{~g} / \mathrm{l}$.

9. Soaking cuttings in liquorice extract at $20 \mathrm{~g} / \mathrm{l}$.

10. Soaking cuttings in yeast extract at $5 \%$.

11 . Soaking cuttings in yeast extract at $10 \%$.

12. Soaking cuttings in algae extract at $2.5 \mathrm{~cm} / 1$.

13. Soaking cuttings in algae extract at $5 \mathrm{~cm} / 1$.

This experiment included 39 combinations which were between 13 dipping and soaking treatments and three sampling dates $(5,6,7$ months after cutting planting).

Picual cuttings of treatments from 1 to 3 were dipped in the growth regulators solution for 5 seconds, while the treatments from 4-13 were soaked in natural extracts for half hour then planted at a depth of five $\mathrm{cm}$ in plastic flats filled with a mixture of silt, peat moss and sand (1: 1: 1 by volume). The flats were kept under tunnel held at green house.

This experiment was used 702 cuttings (6 cuttings $\times 3$ replicates $\times 3$ dates after planting $\times$ 13 treatments) in each season.

The responses of the tested semi-hardwood cuttings Picual olive cv. to the applied dipping and soaking treatments were evaluated through the following parameters:

\section{Rooting characteristics}

Rooting percentage and number of roots/ cutting.

\section{Growth measurements}

Bud sprouting percentage, survival percentage, shoot number/transplant and leaf number/ shoot.

$$
\text { Survival percentage }=\frac{\text { Number of survived cuttings }}{\text { Total number of cuttings }} \times 100
$$

Bud sprouting percentage $=$

Number of sprouted buds on cuttings

Total number of buds on cuttings

\section{Chemical determinations}

\section{Shoot and root carbohydrates percentage}

About $0.2 \mathrm{~g}$ of dry matter samples was added to $50 \mathrm{mM}$ of sulfuric acid $(1 \mathrm{p})$ in Pyrex test tubes $+24 \mathrm{~cm}$ of concentrated sulfuric acid and complement with distilled water. The tubes are placed in a water bath for 3 to 4 hours at a temperature of $70^{\circ} \mathrm{C}$ offset losses with distilled water to maintain the size and focus Nominate standard samples in flasks of $50 \mathrm{~cm}$ and then complement to the mark with distilled water. Then $1 \mathrm{~cm}$ from the filtrate was taken $+1 \mathrm{~cm}$ phenol 5\% (5 g phenol in $10 \mathrm{~cm}$ distilled water) $+5 \mathrm{~cm}$ sulfuric acid. Samples are read on the device Spectrophotometry on the wave length of $490 \mathrm{~nm}$. Compare readings on the device and the corresponding readings were in hard-hit (AOAC, 2012).

\section{Determination of total soluble phenols}

Samples of about $5 \mathrm{~g}$ fresh weights were taken from the shoots and roots at the end of different periods, placed in a glass cup convenient with the addition of $30 \mathrm{~cm}$ alcohol ethyl $80 \%$ to cover the sample surface and heats in water bath at $70^{\circ}$ for 15 minutes. The ethyl extract was transformed into aqueous phase by evaporation at $30 \pm 2{ }^{\circ} \mathrm{C}$ under vacuum for determining total phenols. Folin-Denis Spectrophotometry $(750 \mathrm{mu})$ was used to estimate phenols concentrations as pyrogallol on dry weight basis of cutting tissue $(\mathrm{g} / 100 \mathrm{~g})$ (Cheng and Hanning, 1955).

\section{Statistical Analysis}

The obtained data were statistically analyzed according to the complete randomized block design with 3 replicates each treatment. Data were subjected to analysis of variances (ANOVA) according to Snedecor and Cochran (1990) using the CO-STAT program. Differences between means were compared by using Duncan's multiple range test at 0.05 level (Duncan, 1958).

\section{RESULTS AND DISCUSSION}

\section{Root Characteristics}

\section{Rooting percentage}

Results in Table 1 show that treating with some natural extracts and synthetic growth 
Table 1. Effect of some natural extracts and synthetic growth regulators on rooting percentage of Picual olive cuttings (2014 and 2015 seasons)

\begin{tabular}{|c|c|c|c|c|c|c|c|c|}
\hline \multirow{4}{*}{ Treatment } & \multicolumn{8}{|c|}{ Rooting percentage (\%) } \\
\hline & \multicolumn{4}{|c|}{ First season (2014) } & \multicolumn{4}{|c|}{ Second season (2015) } \\
\hline & \multicolumn{4}{|c|}{ Months after cutting planting } & \multicolumn{4}{|c|}{ Months after cutting planting } \\
\hline & 5 & 6 & 7 & Trea. av. & 5 & 6 & 7 & Trea. av. \\
\hline$\overline{\mathrm{IBA}}$ at $4000 \mathrm{ppm}$ & $88.87 \mathrm{a}$ & $77.73 \mathrm{ab}$ & $77.73 \mathrm{ab}$ & $81.44 \mathrm{~A}$ & $77.73 \mathrm{ab}$ & $77.73 \mathrm{ab}$ & $100.00 \mathrm{a}$ & $85.6 \mathrm{~A}$ \\
\hline NAA at 500 ppm & 44.40cde & $44.40 \mathrm{cde}$ & $55.50 \mathrm{bcd}$ & 48.10 DEF & $55.50 \mathrm{bcd}$ & $55.50 \mathrm{bcd}$ & $44.40 \mathrm{~cd}$ & $51.800 \mathrm{DEF}$ \\
\hline IBA at $4000+\mathrm{NAA}$ at $500 \mathrm{ppm}$ & $66.60 \mathrm{abc}$ & $55.50 \mathrm{bcd}$ & $66.60 \mathrm{abc}$ & $62.90 \mathrm{BCD}$ & $77.73 \mathrm{ab}$ & $77.73 \mathrm{ab}$ & $77.73 \mathrm{ab}$ & $77.73 \mathrm{AB}$ \\
\hline Moringa extr. at $10 \%$ & $11.10 \mathrm{f}$ & $11.10 \mathrm{f}$ & $11.10 \mathrm{f}$ & $11.10 \mathrm{G}$ & $11.10 \mathrm{e}$ & $11.10 \mathrm{e}$ & $11.10 \mathrm{e}$ & $11.10 \mathrm{G}$ \\
\hline Moringa extr. at $20 \%$ & $11.10 \mathrm{f}$ & $22.20 \mathrm{ef}$ & $11.10 \mathrm{f}$ & $14.80 \mathrm{G}$ & $11.10 \mathrm{e}$ & $11.10 \mathrm{e}$ & $11.10 \mathrm{e}$ & $11.10 \mathrm{G}$ \\
\hline Garlic extr.at $10 \%$ & $55.50 \mathrm{bcd}$ & $66.60 \mathrm{abc}$ & $55.50 \mathrm{bcd}$ & 59.20CDE & $55.50 \mathrm{bcd}$ & $55.50 \mathrm{bcd}$ & $55.50 \mathrm{bcd}$ & $55.50 \mathrm{CDE}$ \\
\hline Garlic extr.at $20 \%$ & $55.50 \mathrm{bcd}$ & $177.73 \mathrm{ab}$ & $55.50 \mathrm{bcd}$ & 62.91BCD & $66.60 b c$ & $66.60 \mathrm{bc}$ & 77.73ab & 70.31ABC \\
\hline Liquorice extr.at $5 \mathrm{~g}$ & 44.40cde & $66.60 \mathrm{abc}$ & 44.40 cde & 48.10DEF & $44.40 \mathrm{~cd}$ & $44.40 \mathrm{~cd}$ & $44.40 \mathrm{~cd}$ & $44.40 \mathrm{EF}$ \\
\hline Liquorice extr.at $10 \mathrm{~g}$ & $55.50 \mathrm{bcd}$ & $77.73 \mathrm{ab}$ & $55.50 \mathrm{bcd}$ & $55.50 \mathrm{C}-\mathrm{F}$ & $55.50 \mathrm{bcd}$ & $66.60 \mathrm{bc}$ & $66.63 \mathrm{bc}$ & $62.91 \mathrm{BCD}$ \\
\hline Yeast extr.at $5 \%$ & 33.30def & $44.40 \mathrm{cde}$ & 44.40 cde & $40.70 \mathrm{~F}$ & $44.40 \mathrm{~cd}$ & $33.30 \mathrm{de}$ & 33.30de & $37.00 \mathrm{~F}$ \\
\hline Yeast extr.at $10 \%$ & 44.40cde & $55.50 \mathrm{bcd}$ & 33.30def & $44.40 \mathrm{EF}$ & $44.40 \mathrm{~cd}$ & $44.40 \mathrm{~cd}$ & $44.40 \mathrm{~cd}$ & $44.40 \mathrm{EF}$ \\
\hline Algae extr.at $2.5 \mathrm{~cm} / \mathrm{l}$ & $66.60 \mathrm{abc}$ & $66.63 \mathrm{abc}$ & $77.73 \mathrm{ab}$ & 70.32ABC & $55.50 \mathrm{bcd}$ & $66.60 \mathrm{bc}$ & 77.73ab & 66.61BCD \\
\hline Algae at extr. $5 \mathrm{~cm} / \mathrm{l}$ & 77.73ab & $77.73 \mathrm{ab}$ & $77.73 \mathrm{ab}$ & $77.73 \mathrm{AB}$ & $77.73 \mathrm{ab}$ & $77.73 \mathrm{ab}$ & 77.73ab & $77.73 \mathrm{AB}$ \\
\hline Date av. & $50.39 \mathrm{~A}$ & $54.66 \mathrm{~A}$ & $51.24 \mathrm{~A}$ & & $52.09 \mathrm{~A}$ & $52.95 \mathrm{~A}$ & $55.52 \mathrm{~A}$ & \\
\hline
\end{tabular}

Means in each column which have the same letter(s) are not significantly different.

regulators significantly affected rooting percentage of Picual olive cuttings in the two seasons. However, IBA at $4000 \mathrm{ppm}$ (control treatment) recorded the highest rooting percentage $(81.44$ and $85.60 \%)$ without significant differences with those of algae at 2.5 and $5 \mathrm{~cm}$ treatments $(70.32$ and $77.73 \%)$ in the first season and IBA at $4000 \mathrm{ppm}+\mathrm{NAA}$ at 500 ppm and algae extract at $5 \mathrm{~cm}(77.73 \%)$ in the second one. The lowest rooting percentage was gained by both Moringa extract at 10 and 20\% treatments (from 11.10 to $14.80 \%$ ) in the two seasons. The other soaking treatments (garlic, liquorice and yeast) resulted in between rooting percentages in descending order during the both seasons.

Number of months after planting insignificantly affected rooting percentage in both seasons.

The interaction between dipping treatments and number of months after planting were significant in the two seasons. Higher rooting percentages ( 88.87 and $100.00 \%$ ) were recorded for cuttings dipped in IBA at $4000 \mathrm{ppm}$ after five and seven months in the first and second seasons, respectively compared with those soaked in moringa extract at 10 and $20 \%$ throughout all months after planting in both seasons. The other combinations produced intermediate similar statistically percentages in most cases.

These findings are in agreement with those reported by Aslmoshtaghi and Shahsavar (2010a), Aslmoshtaghi et al. (2014), Khajehpour et al. (2014), Porghorban et al. (2014), Hamooh (2014), Lazaj et al. (2015) and Mohamed (2015). They all reported that IBA increased rooting percentage. Similar trends were confirmed when seaweed extracts was used (Thorsen et al., 2010; Krajnc et al., 2012; Urbanek et al., 2012; Vinoth et al., 2012; 
Lazaj et al., 2015). Németh (2011) stated that, cuttings of clonal mahaleb cultivar 'Egervár' were rooted the best by direct Kelpak treatment.

\section{Number of roots per cutting}

The obtained results in Table 2 reveal that number of roots/cutting was significantly affected by dipping in the natural extracts and synthetic growth regulators in the two seasons. However, IBA at 4000 ppm (14.84 and 14.90 root/cutting) induced the highest number of roots/ cutting, followed by those dipped in IBA $4000 \mathrm{ppm}+$ NAA $500 \mathrm{ppm}$ (11.27 and 11.24 root/ cutting) with significant differences between them in the first and second seasons, respectively. The least number of roots/cutting was recorded for cuttings treated with moringa extract at 10 and $20 \%$ (2.00 and 2.32 and 2.02 and $2.34 \mathrm{root} / \mathrm{cutting}$ ) in the two seasons, respectively. The other treatments tended to a significant decrease in the number of roots/ cutting in both seasons. The number of roots/ cutting generally, ranged from 2.00 to 14.84 root/ cutting in the first season and 2.02 to 14.90 root/ cutting in the second one.

The number of months after cutting planting significantly affected the number of roots/ cutting in both seasons. The highest numbers were recorded for cuttings left until the seventh month after planting ( 8.04 and 8.35 root/cutting) compared with those which were remained for 5 months (5.71 and 5.82 root/cutting) as those gained the lowest numbers in the first and second seasons, respectively.

The interaction between dipping or soaking treatments and number of months after planting was significant throughout the studied seasons, and support the above mentioned trends with regard to the effect of each individual factor on number of roots/cutting. So, the highest number of roots/cutting was recorded with cuttings treated with IBA at $4000 \mathrm{ppm}$ and left until the $7^{\text {th }}, 6^{\text {th }}$ and $5^{\text {th }}$ months from planting (15.80, 15.47 and 13.24 roots in the first season and $16.20,15.19$ and 13.30 roots in the second one), respectively. The lowest root number was resulted from the combinations between moringa extract at 10 and $20 \%$ throughout all months after planting without significant differences between them in both seasons. The other combinations were in-between.
The obtained results are in harmony with those reported by Negash (2003), Sebastiani and Tognetti (2004), El-Ghayaty et al. (2010), Ismaili and Fiku (2010), Aslmoshtaghi and Shahsavar (2010a), Khajehpour et al. (2014), Porghorban et al. (2014), Hamooh (2014), Jan et al. (2014), Lazaj et al. (2015), Mohamed (2015) who reported that using IBA increased number of roots on olive cuttings. Jones and van Staden (1997) cleared that using Kelpak (commercial seaweed) increased rooting percentage and improved rooting quality above that of the control, also, resulting in the development of a vigorous root system consisting of numerous lateral roots. Németh (2011) stated thst, cuttings of clonal mahaleb cultivar 'Egervár' was rooted the best by direct Kelpak (seaweed) treatment.

\section{Vegetative Growth Parameters}

\section{Bud sprouting percentage}

It is clear from results presented in Table 3 that, bud sprouting percentage of Picual olive cuttings was significantly affected by the different investigated treatments in both seasons. The uppermost percentages of bud sprouting were recorded for treatments of IBA at 4000 ppm (48.46 and $48.14 \%$ ), of IBA at $4000+$ NAA at 500 ppm (31.48 and $44.44 \%$ ), garlic extract at $20 \%$ (28.72 and $44.42 \%$ ) and liquorice extract at $10 \mathrm{~g}$ (31.48 and 43.04\%) without significant differences between them in both seasons. The differences between treatments of algae extract at $5 \mathrm{~cm}$, garlic extract at $20 \%$ and liquorice extract at $10 \mathrm{~g}$ were insignificant in both seasons. Moringa extract and yeast treatments recorded the lowest percentages.

The results cleared also that bud sprouting percentage in Picual olive cuttings were significantly differed by the various dates after cutting planting in both seasons. The highest values of bud sprouting percentage (33.40 and $33.55 \%$ ) were detected after seven month from planting in both seasons. The least sprouting percentage was recorded after the fifth month from planting (23.08 and $22.12 \%)$ in the two seasons.

The interaction between treatments and number of months after planting on bud sprouting percentage was significant in both 
Table 2. Effect of some natural extracts and synthetic growth regulators on root number / cutting of Picual olive variety (2014 and 2015 seasons)

\begin{tabular}{|c|c|c|c|c|c|c|c|c|}
\hline \multirow[t]{4}{*}{ Treatment } & \multicolumn{8}{|c|}{ Number of roots/ cutting } \\
\hline & & First seas & $\operatorname{son}(2014)$ & & \multirow{2}{*}{\multicolumn{4}{|c|}{$\begin{array}{c}\text { Second season (2015) } \\
\text { Months after cutting planting }\end{array}$}} \\
\hline & \multicolumn{4}{|c|}{ Months after cutting planting } & & & & \\
\hline & 5 & 6 & 7 & Trea. av. & 5 & $\overline{6}$ & 7 & Trea. av. \\
\hline IBA at $4000 \mathrm{ppm}$ & $13.24 a b c$ & $15.47 \mathrm{ab}$ & $15.80 \mathrm{a}$ & $14.84 \mathrm{~A}$ & $13.30 \mathrm{bc}$ & $15.19 \mathrm{ab}$ & $16.20 \mathrm{a}$ & $14.90 \mathrm{~A}$ \\
\hline NAA at 500 ppm & $7.80 \mathrm{f}-\mathrm{j}$ & $8.81 \mathrm{e}-\mathrm{h}$ & 9.74def & $8.78 \mathrm{CD}$ & $7.13 \mathrm{~h}-\mathrm{n}$ & $9.10 \mathrm{~d}-\mathrm{h}$ & $10.03 \mathrm{~d}-\mathrm{g}$ & $8.76 \mathrm{CD}$ \\
\hline IBA at $4000+N A A$ at $500 \mathrm{ppm}$ & $9.34 \mathrm{~d}-\mathrm{g}$ & $11.70 \mathrm{~cd}$ & $12.78 \mathrm{bc}$ & $11.27 \mathrm{~B}$ & $9.33 \mathrm{~d}-\mathrm{h}$ & 11.10 cde & $13.278 \mathrm{bc}$ & $11.24 \mathrm{~B}$ \\
\hline Moringa extr. at $10 \%$ & $1.47 \mathrm{~s}$ & $2.07 \mathrm{rs}$ & $2.47 \mathrm{qrs}$ & $2.00 \mathrm{H}$ & $1.53 \mathrm{~s}$ & $1.90 \mathrm{rs}$ & $2.63 \mathrm{qrs}$ & $2.02 \mathrm{G}$ \\
\hline Moringa extr. at $20 \%$ & $1.77 \mathrm{~s}$ & 2.30rs & $2.90 \mathrm{p}-\mathrm{s}$ & $2.32 \mathrm{GH}$ & $2.10 \mathrm{rs}$ & $2.23 \mathrm{rs}$ & $2.70 \mathrm{qrs}$ & $2.34 \mathrm{G}$ \\
\hline Garlic extr.at $10 \%$ & $4.70 \mathrm{~m}-\mathrm{r}$ & $7.10 \mathrm{f}-\mathrm{m}$ & $7.51 \mathrm{f}-1$ & $6.44 \mathrm{E}$ & $4.33 \mathrm{n}-\mathrm{s}$ & $7.23 \mathrm{~g}-\mathrm{m}$ & $7.90 \mathrm{f}-\mathrm{j}$ & $6.49 \mathrm{E}$ \\
\hline Garlic extr.at $20 \%$ & $5.18 \mathrm{j}-\mathrm{q}$ & $7.21 \mathrm{f}-\mathrm{m}$ & $7.97 \mathrm{f}-\mathrm{j}$ & $6.79 \mathrm{E}$ & $5.20 \mathrm{j}-\mathrm{q}$ & $7.40 \mathrm{f}-1$ & 8.47 e-i & $7.02 \mathrm{E}$ \\
\hline Liquorice extr.at $5 \mathrm{~g}$ & $4.81 \mathrm{k}-\mathrm{r}$ & $6.50 \mathrm{~h}-\mathrm{o}$ & $7.57 \mathrm{f}-\mathrm{k}$ & $6.29 \mathrm{E}$ & $5.07 \mathrm{k}-\mathrm{q}$ & $6.83 \mathrm{~h}-\mathrm{o}$ & $7.90 \mathrm{f}-\mathrm{j}$ & $6.60 \mathrm{E}$ \\
\hline Liquorice extr.at10 g & 5.65 i-p & $7.45 \mathrm{f}-\mathrm{m}$ & 8.45 e-i & $7.18 \mathrm{DE}$ & 5.73 i-p & $7.63 \mathrm{f}-\mathrm{k}$ & 8.50 e-i & $7.29 \mathrm{DE}$ \\
\hline Yeast extr.at $5 \%$ & $2.78 \mathrm{qrs}$ & $3.83 \mathrm{o}-\mathrm{s}$ & $4.721-\mathrm{r}$ & $3.78 \mathrm{FG}$ & $3.23 \mathrm{p}-\mathrm{s}$ & $4.03 \mathrm{o}-\mathrm{s}$ & $4.701-r$ & $3.99 \mathrm{~F}$ \\
\hline Yeast extr.at $10 \%$ & $3.23 \mathrm{p}-\mathrm{s}$ & $4.13 \mathrm{n}-\mathrm{s}$ & $4.64 \mathrm{~m}-\mathrm{r}$ & $4.00 \mathrm{~F}$ & $3.43 \mathrm{p}-\mathrm{s}$ & $4.53 \mathrm{~m}-\mathrm{r}$ & $5.10 \mathrm{j}-\mathrm{q}$ & $4.36 \mathrm{~F}$ \\
\hline Algae extr.at $2.5 \mathrm{~cm} / \mathrm{l}$ & $6.78 \mathrm{~g}-\mathrm{n}$ & $7.18 \mathrm{f}-\mathrm{m}$ & $9.17 \mathrm{~d}-\mathrm{h}$ & 7.71CDE & $6.70 \mathrm{~h}-\mathrm{o}$ & $7.40 \mathrm{f}-1$ & $9.40 \mathrm{~d}-\mathrm{h}$ & $7.83 \mathrm{DE}$ \\
\hline Algae at extr. $5 \mathrm{~cm} / \mathrm{l}$ & $7.51 f-1$ & $9.53 \mathrm{~d}-\mathrm{g}$ & $10.81 \mathrm{cde}$ & $9.28 \mathrm{C}$ & $8.53 \mathrm{e}-\mathrm{i}$ & $10.17 \mathrm{def}$ & $11.80 \mathrm{~cd}$ & $10.17 \mathrm{BC}$ \\
\hline Date av. & $5.71 \mathrm{C}$ & $7.18 \mathrm{~B}$ & $8.04 \mathrm{~A}$ & & $5.82 \mathrm{C}$ & $7.29 \mathrm{~B}$ & $8.35 \mathrm{~A}$ & \\
\hline
\end{tabular}

Means in each column which have the same letter(s) are not significantly different.

Table 3. Effect of some natural extracts and synthetic growth regulators on sprouting percentage of Picual olive cuttings (2014 and 2015 seasons)

\begin{tabular}{|c|c|c|c|c|c|c|c|c|}
\hline \multirow[t]{4}{*}{ Treatment } & \multicolumn{8}{|c|}{ Percentage of sprouting (\%) } \\
\hline & \multicolumn{4}{|c|}{ First season (2014) } & \multicolumn{4}{|c|}{ Second season (2015) } \\
\hline & \multicolumn{4}{|c|}{ Months after cutting planting } & \multicolumn{4}{|c|}{ Months after cutting planting } \\
\hline & 5 & 6 & 7 & Trea. av. & 5 & 6 & 7 & Trea. av. \\
\hline IBA at $4000 \mathrm{ppm}$ & $38.90 \mathrm{a}-\mathrm{f}$ & $52.80 \mathrm{ab}$ & $53.67 \mathrm{a}$ & $48.46 \mathrm{~A}$ & $41.67 \mathrm{~b}-\mathrm{f}$ & $44.43 \mathrm{a}-\mathrm{e}$ & $58.33 \mathrm{ab}$ & $48.14 \mathrm{~A}$ \\
\hline NAA at $500 \mathrm{ppm}$ & $13.89 \mathrm{~g}-\mathrm{k}$ & $33.33 \mathrm{~b}-\mathrm{g}$ & $30.57 \mathrm{c}-\mathrm{h}$ & $25.93 \mathrm{CD}$ & $27.77 \mathrm{e}-\mathrm{j}$ & $37.50 \mathrm{c}-\mathrm{g}$ & $19.47 \mathrm{~g}-1$ & $28.24 \mathrm{CD}$ \\
\hline IBA at $4000+\mathrm{NAA}$ at $500 \mathrm{ppm}$ & $33.30 \mathrm{~b}-\mathrm{g}$ & $19.45 \mathrm{f}-\mathrm{k}$ & $41.70 \mathrm{a}-\mathrm{e}$ & $31.48 \mathrm{BCD}$ & $33.33 d-h$ & 44.43 a-e & $55.57 \mathrm{abc}$ & $44.44 \mathrm{AB}$ \\
\hline Moringa extr. at $10 \%$ & $5.57 \mathrm{jk}$ & $5.57 \mathrm{jk}$ & $8.33 \mathrm{ijk}$ & $6.49 \mathrm{E}$ & $16.68 \mathrm{~h}-1$ & $8.33 \mathrm{kl}$ & $8.33 \mathrm{kl}$ & $11.11 \mathrm{~F}$ \\
\hline Moringa extr. at $20 \%$ & $2.78 \mathrm{k}$ & $5.57 \mathrm{jk}$ & $11.10 \mathrm{~h}-\mathrm{k}$ & $6.48 \mathrm{E}$ & $12.51 \mathrm{i}-1$ & 5.531 & $8.33 \mathrm{kl}$ & $8.79 \mathrm{~F}$ \\
\hline Garlic extr. at $10 \%$ & $8.33 \mathrm{ijk}$ & $27.77 \mathrm{c}-\mathrm{i}$ & $45.83 \mathrm{abc}$ & 27.31BCD & $25.00 \mathrm{f}-\mathrm{k}$ & $41.67 \mathrm{~b}-\mathrm{f}$ & $41.64 b-f$ & $36.10 \mathrm{BC}$ \\
\hline Garlic extr. at $20 \%$ & $25.00 \mathrm{~d}-\mathrm{j}$ & $16.70 \mathrm{~g}-\mathrm{k}$ & $44.47 \mathrm{a}-\mathrm{d}$ & $28.72 \mathrm{BCD}$ & 30.53 e-i & $58.30 \mathrm{ab}$ & $44.43 \mathrm{a}-\mathrm{e}$ & $44.42 \mathrm{AB}$ \\
\hline Liquorice extr. at 5g/l & $22.24 \mathrm{e}-\mathrm{k}$ & $16.65 \mathrm{~g}-\mathrm{k}$ & $20.83 \mathrm{f}-\mathrm{k}$ & $19.91 \mathrm{D}$ & $25.00 \mathrm{f}-\mathrm{k}$ & $19.44 \mathrm{~g}-1$ & $25.00 \mathrm{f}-\mathrm{k}$ & $23.15 \mathrm{DE}$ \\
\hline Liquorice extr.at10 g/l & $25.00 \mathrm{~d}-\mathrm{j}$ & $30.57 \mathrm{c}-\mathrm{h}$ & $38.87 \mathrm{a}-\mathrm{f}$ & $31.48 \mathrm{BCD}$ & 29.17 e-j & $38.87 \mathrm{c}-\mathrm{f}$ & $61.10 \mathrm{a}$ & $43.04 \mathrm{AB}$ \\
\hline Yeast extr.at $5 \%$ & $30.57 \mathrm{c}-\mathrm{h}$ & $33.30 \mathrm{~b}-\mathrm{g}$ & $45.83 \mathrm{abc}$ & $36.57 \mathrm{BC}$ & $12.51 \mathrm{i}-1$ & $11.12 \mathrm{jkl}$ & $14.07 \mathrm{i}-1$ & $12.57 \mathrm{EF}$ \\
\hline Yeast extr.at $10 \%$ & $25.01 \mathrm{~d}-\mathrm{j}$ & $16.65 \mathrm{~g}-\mathrm{k}$ & $33.30 \mathrm{~b}-\mathrm{g}$ & $24.99 \mathrm{CD}$ & $8.33 \mathrm{kl}$ & $11.12 \mathrm{jkl}$ & $19.44 \mathrm{~g}-1$ & $12.96 \mathrm{EF}$ \\
\hline Algae extr.at $2.5 \mathrm{~cm} / \mathrm{l}$ & $25.00 \mathrm{~d}-\mathrm{j}$ & $37.57 \mathrm{a}-\mathrm{f}$ & $20.83 \mathrm{f}-\mathrm{k}$ & $27.80 \mathrm{BCD}$ & $11.12 \mathrm{jkl}$ & $41.67 \mathrm{~b}-\mathrm{f}$ & $30.57 \mathrm{e}-\mathrm{i}$ & $27.78 \mathrm{CD}$ \\
\hline Algae at extr. $5 \mathrm{~cm} / \mathrm{l}$ & $44.47 \mathrm{a}-\mathrm{d}$ & $33.33 \mathrm{~b}-\mathrm{g}$ & $38.88 \mathrm{a}-\mathrm{f}$ & $38.89 \mathrm{AB}$ & $13.89 \mathrm{i}-1$ & 44.47 a-e & $49.87 \mathrm{a}-\mathrm{d}$ & $36.07 \mathrm{BC}$ \\
\hline Date av. & $23.08 \mathrm{~B}$ & 25.33B & $33.40 \mathrm{~A}$ & & $22.12 \mathrm{~B}$ & $31.30 \mathrm{~A}$ & $33.55 \mathrm{~A}$ & \\
\hline
\end{tabular}

Means in each column which have the same letter(s) are not significantly different. 
seasons. The highest percentage of bud sprouting was detected from each of the following combinations IBA at $4000 \mathrm{ppm}$, IBA at $4000 \mathrm{ppm}+\mathrm{NAA}$ at $500 \mathrm{ppm}$ and liquorice extract at $10 \mathrm{~g}$ after 7 month from planting. In addition, the combination of garlic extract at $20 \%$ after 6 month from planting and algae extract at $5 \mathrm{~cm}$ after 7 months from planting gained the percentages of bud sprouting (58.30 and $49.87 \%$ ) in the second season.

The obtained results are in line with those reported by Patil et al. (2001) Mayer et al. (2013) and Gill et al. (2014) who reported that used IBA improved sprouting percentage.

\section{Number of shoots / transplant}

Results presented in Table 4 reveal significant differences among the different treatments in both seasons. The highest shoot number/ transplant was obtained from each of the following treatments, IBA at $4000 \mathrm{ppm}(2.74$ and 2.17 shoot/transplant), IBA at $4000 \mathrm{ppm}+$ NAA at 500 ppm $(2.01 ; 2.39$ shoot/ transplant $)$, garlic extract at 10 and $20 \%(2.10 ; 2.20$ and $2.33 ; 2.73$ shoot/transplant), liquorice extract at $10 \mathrm{~g} \mathrm{(2.09}$ and 2.11 shoot/transplant) and algae extract at $5 \mathrm{~cm}$ (2.38 and 2.29 shoot/transplant) in the $1^{\text {st }}$ and $2^{\text {nd }}$ season, respectively. The lowest numbers of shoot / transplant were recorded for the two concentrations of moringa extract (10 and 20\%) and yeast extract (5 and $10 \%$ ) in both seasons without significant differences between the two concentrations.

Results clarified significant differences between the dates after planting on number of the shoots per transplant in the two seasons. The optimum number of shoots / transplant (2.16 and 2.16 shoot/transplant) was traced seven months after planting in both seasons. The lowest number of shoots/ transplant (1.73 and 1.45 shoot/transplant) was found fifth month after planting in both seasons. Insignificant differences between 5 and 6 months in the first season and likewise, between 6 and 7 months in the second season were traced.

The interaction between treatments and number of months after planting on number of shoots per cutting was significant in both seasons. The highest number of shoots/cutting were recorded for IBA at $4000 \mathrm{ppm}+\mathrm{NAA}$ at
$500 \mathrm{ppm}$ treatment after 7 months, garlic at 20 after 6 and 7 months and algae at $5 \mathrm{~cm}$ after 7 months from planting in the two seasons .

Similar trends were reported by Martins $\boldsymbol{e t}$ al. (2001), Patil et al.( 2001), Da Silva et al. (2013), Khajehpour et al. (2014), Sharma et al. (2014), Jan et al. (2014) and Jana et al. (2015). They all cleared that IBA increased shoot number / cutting of fruit species.

The increase in shoot characteristics might also be due to the auxins content in the seaweed extracts which have an effective role in cell division and enlargement; this leads to increase the shoot growth, leaf area and plant dry weight (Gollan and Wright, 2006). Arthur et al. (2003) showed that seaweed improves rootgrowth, vegetative and reproductive growth of many plants. Mansour et al. (2006) and Abd El-Moniem and Abd-Allah (2008) showed that the applied of algae extract was very effective in stimulating the growth characters.

At contrast, Sadak (2016) reported that yeast extract caused significant decreases in number of shoot of Pisum sativum L. plant.

\section{Leaf number/shoot}

As shown in Table 5 the effect of the tested treatments on number of leaves/ cutting was significant in the two seasons. The highest leaf number per cutting was recorded for each of the following treatment, IBA at $4000 \mathrm{ppm}$ (6. 49 and 6.61), IBA at $4000 \mathrm{ppm}+\mathrm{NAA}$ at $500 \mathrm{ppm}$ (5.85 and 5.80), liquorice $10 \mathrm{~g}$ (6.01 and 5.48) and algae $5 \mathrm{~cm}(6.29$ and 6.16$)$ in the two seasons, respectively without differences between most of them. The differences between the two concentrations of moringa, yeast and algae on number of leaves per cutting were insignificant in the two seasons. The lowest numbers of shoots/plant were gained the two concentrations of moringa $(1.08,0.98$ and $1.21,1.17)$ in both seasons, respectively without significan differences between them.

Also, the effect of months after planting on number of leaves/ cutting was significant in the two seasons. The highest number of leaves/ cutting (5.97 and 6.02 leaves/ cutting) was found seven months after planting in both seasons. The lowest number of leaves/ cutting (4.21 and 3.36 leaves/ cutting) was traced 5 months after planting in both seasons. 
Table 4. Effect of some natural extracts and synthetic growth regulators on shoot number/ cutting of Picual olive variety (2014 and 2015 seasons)

\begin{tabular}{|c|c|c|c|c|c|c|c|c|}
\hline \multirow[t]{4}{*}{ Treatment } & \multicolumn{8}{|c|}{ Shoot number/ transplant } \\
\hline & \multicolumn{4}{|c|}{ First season (2014) } & \multicolumn{4}{|c|}{ Second season (2015) } \\
\hline & \multicolumn{4}{|c|}{ Months after cutting planting } & \multicolumn{4}{|c|}{ Months after cutting planting } \\
\hline & $\overline{5}$ & $\overline{6}$ & 7 & Trea. av. & 5 & $\overline{6}$ & 7 & Trea. av. \\
\hline$\overline{\text { IBA at } 4000 \mathrm{ppm}}$ & $2.20 \mathrm{a}-\mathrm{g}$ & $3.12 \mathrm{a}$ & $2.91 \mathrm{ab}$ & $2.74 \mathrm{~A}$ & $1.87 \mathrm{c}-\mathrm{i}$ & $2.43 \mathrm{a}-\mathrm{g}$ & $2.20 \mathrm{~b}-\mathrm{h}$ & $2.17 \mathrm{~B}-\mathrm{E}$ \\
\hline NAA at $500 \mathrm{ppm}$ & $1.58 \mathrm{e}-\mathrm{j}$ & $2.24 \mathrm{a}-\mathrm{g}$ & $2.24 \mathrm{a}-\mathrm{g}$ & $2.02 \mathrm{BC}$ & $1.33 \mathrm{~h}-\mathrm{m}$ & $2.15 b-h$ & $1.80 \mathrm{c}-\mathrm{i}$ & $1.76 \mathrm{DE}$ \\
\hline IBA at $4000+\mathrm{NAA}$ at $500 \mathrm{ppm}$ & $1.79 \mathrm{~d}-\mathrm{i}$ & $1.66 \mathrm{~d}-\mathrm{i}$ & $2.58 \mathrm{a}-\mathrm{e}$ & $2.01 \mathrm{BC}$ & $1.63 \mathrm{e}-\mathrm{k}$ & $2.43 \mathrm{a}-\mathrm{g}$ & $3.10 \mathrm{ab}$ & $2.39 \mathrm{AB}$ \\
\hline Moringa extr. at $10 \%$ & $0.44 \mathrm{k}$ & $0.57 \mathrm{jk}$ & $0.90 \mathrm{~h}-\mathrm{k}$ & $0.64 \mathrm{D}$ & $0.43 \mathrm{~m}$ & $0.57 \mathrm{~lm}$ & $0.67 \mathrm{klm}$ & $0.56 \mathrm{~F}$ \\
\hline Moringa extr. at $20 \%$ & $0.57 \mathrm{jk}$ & $0.77 \mathrm{ijk}$ & $0.90 \mathrm{~h}-\mathrm{k}$ & $0.74 \mathrm{D}$ & $0.43 \mathrm{~m}$ & $0.57 \mathrm{~lm}$ & $0.77 \mathrm{j}-\mathrm{m}$ & $0.59 \mathrm{~F}$ \\
\hline Garlic extr. at $10 \%$ & $1.33 \mathrm{~g}-\mathrm{k}$ & 2.12a-g & $2.85 \mathrm{abc}$ & $2.10 \mathrm{BC}$ & $1.80 \mathrm{c}-\mathrm{i}$ & $2.43 \mathrm{a}-\mathrm{g}$ & $2.77 \mathrm{abc}$ & $2.33 \mathrm{ABC}$ \\
\hline Garlic extr. at $20 \%$ & $1.80 \mathrm{c}-\mathrm{i}$ & 2.23a-g & $2.58 \mathrm{a}-\mathrm{e}$ & $2.20 \mathrm{ABC}$ & $2.20 \mathrm{~b}-\mathrm{h}$ & 2.73abc & $3.27 \mathrm{a}$ & $2.73 \mathrm{~A}$ \\
\hline Liquorice extr. at 5g/l & $1.91 \mathrm{~b}-\mathrm{h}$ & 2.16a-g & $2.10 \mathrm{a}-\mathrm{g}$ & $2.06 \mathrm{BC}$ & $2.00 \mathrm{c}-\mathrm{i}$ & $1.43 \mathrm{~h}-1$ & $2.00 \mathrm{c}-\mathrm{i}$ & $1.81 \mathrm{CDE}$ \\
\hline Liquorice extr.at10 g/l & $2.34 \mathrm{a}-\mathrm{g}$ & $1.68 \mathrm{~d}-\mathrm{i}$ & $2.24 \mathrm{a}-\mathrm{g}$ & $2.09 \mathrm{BC}$ & $1.57 \mathrm{f}-\mathrm{k}$ & $2.20 \mathrm{~b}-\mathrm{h}$ & $2.57 \mathrm{a}-\mathrm{e}$ & 2.11B-E \\
\hline Yeast extr.at $5 \%$ & $2.16 \mathrm{a}-\mathrm{g}$ & $2.10 \mathrm{a}-\mathrm{g}$ & $2.66 a-d$ & $2.31 \mathrm{ABC}$ & $1.35 \mathrm{~h}-\mathrm{m}$ & $1.50 \mathrm{~g}-1$ & $2.00 \mathrm{c}-\mathrm{i}$ & $1.62 \mathrm{E}$ \\
\hline Yeast extr.at $10 \%$ & $1.90 \mathrm{~b}-\mathrm{h}$ & $1.51 \mathrm{f}-\mathrm{j}$ & $1.85 \mathrm{c}-\mathrm{h}$ & $1.75 \mathrm{C}$ & $1.15 \mathrm{i}-\mathrm{m}$ & $1.70 \mathrm{~d}-\mathrm{j}$ & $2.00 \mathrm{c}-\mathrm{i}$ & $1.62 \mathrm{E}$ \\
\hline Algae extr.at $2.5 \mathrm{~cm} / \mathrm{l}$ & $1.91 \mathrm{~b}-\mathrm{h}$ & $1.90 \mathrm{~b}-\mathrm{h}$ & $1.85 \mathrm{~b}-\mathrm{h}$ & $1.89 \mathrm{BC}$ & $1.43 \mathrm{~h}-1$ & $2.20 \mathrm{~b}-\mathrm{h}$ & $2.23 \mathrm{~b}-\mathrm{h}$ & 1.96B-E \\
\hline Algae at extr. $5 \mathrm{~cm} / \mathrm{l}$ & $2.57 \mathrm{a}-\mathrm{f}$ & $2.13 \mathrm{a}-\mathrm{g}$ & $2.45 \mathrm{a}-\mathrm{f}$ & $2.38 \mathrm{AB}$ & $1.67 \mathrm{e}-\mathrm{j}$ & $2.53 \mathrm{a}-\mathrm{f}$ & $2.67 \mathrm{a}-\mathrm{d}$ & 2.29A-D \\
\hline Date av. & $1.73 \mathrm{~B}$ & $1.86 \mathrm{~B}$ & $2.16 \mathrm{~A}$ & & $1.45 \mathrm{~B}$ & $1.91 \mathrm{~A}$ & $2.16 \mathrm{~A}$ & \\
\hline
\end{tabular}

Means in each column which have the same letter(s) are not significantly different.

Table 5. Effect of some natural extracts and synthetic growth regulators on leaf number/ shoot of Picual olive variety (2014 and 2015 seasons)

\begin{tabular}{|c|c|c|c|c|c|c|c|c|}
\hline \multirow[t]{4}{*}{ Treatment } & \multicolumn{8}{|c|}{ Leaf number/shoot } \\
\hline & \multicolumn{4}{|c|}{ First season (2014) } & \multicolumn{4}{|c|}{ Second season (2015) } \\
\hline & \multicolumn{4}{|c|}{ Months after cutting planting } & \multicolumn{4}{|c|}{ Months after cutting planting } \\
\hline & 5 & 6 & 7 & Trea. av. & 5 & 6 & $\overline{7}$ & Trea. av. \\
\hline$\overline{\text { IBA at } 4000 \mathrm{ppm}}$ & $5.49 \mathrm{~b}-\mathrm{i}$ & $5.67 \mathrm{~b}-\mathrm{i}$ & $8.32 \mathrm{a}$ & $6.49 \mathrm{~A}$ & $5.13 \mathrm{~g}-\mathrm{n}$ & $6.82 \mathrm{~b}-\mathrm{f}$ & $7.89 \mathrm{ab}$ & $\overline{6.61 \mathrm{AB}}$ \\
\hline NAA at $500 \mathrm{ppm}$ & 4.13hij & $3.03 \mathrm{jk}$ & $5.80 \mathrm{~b}-\mathrm{h}$ & $4.32 \mathrm{D}$ & $3.53 \mathrm{n}-\mathrm{q}$ & $5.12 \mathrm{~g}-\mathrm{n}$ & $5.62 \mathrm{~d}-\mathrm{k}$ & $4.76 \mathrm{DE}$ \\
\hline IBA at $4000+\mathrm{NAA}$ at $500 \mathrm{ppm}$ & 4.45hij & $6.53 \mathrm{a}-\mathrm{g}$ & $6.55 \mathrm{a}-\mathrm{g}$ & $5.85 \mathrm{AB}$ & $3.75 \mathrm{~m}-\mathrm{q}$ & $6.75 \mathrm{~b}-\mathrm{g}$ & $6.91 \mathrm{~b}-\mathrm{e}$ & $5.80 \mathrm{BC}$ \\
\hline Moringa extr. at $10 \%$ & 0.891 & 1.071 & $1.30 \mathrm{kl}$ & $1.08 \mathrm{E}$ & $0.75 \mathrm{r}$ & $1.07 \mathrm{r}$ & $1.13 \mathrm{r}$ & $0.98 \mathrm{G}$ \\
\hline Moringa extr. at 20\% & 0.871 & $1.27 \mathrm{kl}$ & $1.50 \mathrm{kl}$ & $1.21 \mathrm{E}$ & $0.89 \mathrm{r}$ & $1.22 \mathrm{r}$ & $1.40 \mathrm{r}$ & $1.17 \mathrm{G}$ \\
\hline Garlic extr. at $10 \%$ & $3.80 \mathrm{ij}$ & 4.1000 hij & $6.74 \mathrm{a}-\mathrm{f} 4$ & $4.88 \mathrm{BCD}$ & $3.28 \mathrm{pq}$ & $5.53 \mathrm{e}-1$ & 7.61ab & $5.48 \mathrm{CD}$ \\
\hline Garlic extr. at $20 \%$ & $5.14 \mathrm{~d}-\mathrm{i}$ & $4.89 \mathrm{f}-\mathrm{j}$ & 6.93 a-e & $5.65 \mathrm{AB}$ & $3.37 \mathrm{opq}$ & $5.00 \mathrm{~h}-\mathrm{o}$ & 7.42abc & $5.26 \mathrm{CD}$ \\
\hline Liquorice extr. at $5 \mathrm{~g} / \mathrm{l}$ & 4.53hij & $4.83 \mathrm{f}-\mathrm{j}$ & $7.16 \mathrm{abc} 5$ & $5.51 \mathrm{ABC}$ & 3.931-p & $4.63 j-p$ & $6.45 \mathrm{~b}-\mathrm{i}$ & 5.01 CDE \\
\hline Liquorice extr.at10 g/l & $5.25 \mathrm{c}-\mathrm{i}$ & $5.55 \mathrm{~b}-\mathrm{i}$ & $7.23 \mathrm{ab}$ & $6.01 \mathrm{~A}$ & $3.94 \mathrm{k}-\mathrm{p}$ & $5.92 \mathrm{c}-\mathrm{j}$ & $6.58 \mathrm{~b}-\mathrm{h}$ & $5.48 \mathrm{CD}$ \\
\hline Yeast extr.at $5 \%$ & $4.64 \mathrm{~g}-\mathrm{j}$ & $3.83 \mathrm{ij}$ & $5.08 \mathrm{e}-\mathrm{i}$ & $4.52 \mathrm{CD}$ & $2.20 \mathrm{qr}$ & $3.97 \mathrm{k-p}$ & $5.18 \mathrm{f}-\mathrm{n}$ & $3.78 \mathrm{~F}$ \\
\hline Yeast extr.at $10 \%$ & $5.03 \mathrm{e}-\mathrm{i}$ & $5.57 \mathrm{~b}-\mathrm{i}$ & $5.80 \mathrm{~b}-\mathrm{h} 5$ & $5.47 \mathrm{ABC}$ & $3.38 \mathrm{opq}$ & $3.77 \mathrm{~m}-\mathrm{q}$ & $5.24 \mathrm{e}-\mathrm{m}$ & $4.13 \mathrm{EF}$ \\
\hline Algae extr.at $2.5 \mathrm{~cm} / \mathrm{l}$ & $5.12 \mathrm{~d}-\mathrm{i}$ & $5.66 \mathrm{~b}-\mathrm{i}$ & $7.00 \mathrm{a}-\mathrm{d}$ & $5.93 \mathrm{AB}$ & $4.60 \mathrm{j}-\mathrm{p}$ & $7.25 \mathrm{a}-\mathrm{d}$ & $8.03 \mathrm{ab}$ & $6.63 \mathrm{AB}$ \\
\hline Algae at extr. $5 \mathrm{~cm} / \mathrm{l}$ & $5.44 \mathrm{~b}-\mathrm{i}$ & $5.31 \mathrm{c}-\mathrm{i}$ & $8.13 \mathrm{a}$ & $6.29 \mathrm{~A}$ & 4.87i-p & $7.78 \mathrm{ab}$ & $8.82 \mathrm{a}$ & $7.16 \mathrm{~A}$ \\
\hline Date av. & $4.21 \mathrm{~B}$ & $4.41 \mathrm{~B}$ & $5.97 \mathrm{~A}$ & & $3.36 \mathrm{C}$ & $4.99 \mathrm{~B}$ & $6.02 \mathrm{~A}$ & \\
\hline
\end{tabular}

Means in each column which have the same letter(s) are not significantly different. 
The interaction between treatments and number of months after planting on leaf number per cutting was significant in both seasons. The highest number of leaves/cutting was recorded for each of the combinations IBA at $4000 \mathrm{ppm}$, IBA at $4000 \mathrm{ppm}+\mathrm{NAA}$ at $500 \mathrm{ppm}$, garlic, liquorice and algae 7 months after planting without significant differences between them in the two seasons. All combinations of moringa levels at different dates gave the lowest number of leaves /cutting in both seasons.

These findings are entirely in agreement with those reported by Patil et al. (2001), Sharma et al. (2014) and Jan et al. (2014) reported that IBA increased leaf number/cutting of fruit species (olive, pear, apple and grapvine). On the other hand, IBA did not significantly affect on leaf number/cutting (Dolor et al., 2010).

Mansour et al. (2006), Abd El-Moniem and Abd-Allah (2008), Vinoth et al. (2012), Abdulrahman (2013) and Ibrahim (2013) reported that the applied of algae extract was very effective in stimulating the growth characters (i.e., leaf number/cutting) of olive cv. Hojblanca. Babilie et al. (2015) mentioned that licorice roots and seaweed extracts significantly increased plant height, length of the tallest leaf and number.

Zaki and Rady (2015) showed that the Moringa oleifera leaf extract (MLE) significantly increased growth characteristics (i.e., shoot length, number and area of leaves per plant, and plant dry weight) when compared with the controls.

Taha et al. (2016) cleared that yeast extract had no significant effect on stem diameter, number of leaves/plant and root length of Azadirachta indica plants. On the other hand, Sadak (2016) mentioned that yeast extract caused significant decreases in number of leaves of Pisum sativum L. plants.

\section{Survival percentage of cuttings}

Results in Table 6 indicate that, the effect of the studied treatments on survival percentage of cuttings was significant in the two seasons. The highest survival percentage was recorded for each of the following treatments IBA at 4000 ppm and NAA at $500 \mathrm{ppm}$, garlic at 10 and 20, liquorice extract at $10 \mathrm{~g}$ and algae at 2.5 and 5 $\mathrm{cm}$ in both seasons without significant differences between them. The least survival percentage was gained by the two concentrations of moringa extract at 10\% (11.10 and $22.20 \%$ ) and $20 \%$ (11.10 and 29.60\%) in both seasons without significant differences between the two concentrations in both seasons.

The effect of different months after planting on the survival percentage of cuttings was significantly differed in both seasons. The maximum survival percentages were traced fife and six months after planting $(69.30,57.22 \%$ and $75.18,57.65 \%$ ) in the first and second season, respectively. The differences between survival percentage of cuttings after 6 and 7 months were insignificant in the two seasons.

The interaction between treatments and number of months after planting on survival percentage was significant in the two seasons. All combinations of synthetic growth regulators, garlic levels, liquorice extract at $10 \mathrm{~g}$ and algae extract after the different dates gave the highest survival percentage of cuttings without significant differences between them in both seasons.

Similar trends were reported by Martins $\boldsymbol{e t}$ al. (2001), Patil et al. (2001), Andrade and Martins (2003), Jiang et al. (2004), Samaan et al. (2010), Agele et al. (2013), Mayer et al. (2014), Jan et al. (2014) and Soni et al. (2016). They all reported that IBA increased survival percentage of cuttings in different fruit species (olive, pear, apple, peach, plum, citrus and grapevine).

Vinoth et al. (2012) mentioned that the application of algae extract was very effective in stimulating growth characters i.e., survival percentage of cuttings. Arthur et al. (2003) showed that seaweed increases seedling quality and survival, improves root-growth, vegetative and reproductive growth of many plants.

\section{Chemical Constituents in Shoots and Roots of Survived Cutting}

\section{Total carbohydrates percentage}

The results presented in Table 7 show that the highest content of total carbohydrates in roots of cuttings treated with IBA at $4000 \mathrm{ppm}+\mathrm{NAA}$ at $500 \mathrm{ppm}$ (13.20 and 13.34\%) in both seasons, 
Table 6. Effect of some natural extracts and synthetic growth regulators on survival percentage of Picual olive cuttings (2014 and 2015 seasons)

\begin{tabular}{|c|c|c|c|c|c|c|c|c|}
\hline \multirow[t]{4}{*}{ Treatment } & \multicolumn{8}{|c|}{ Survival percentage (\%) } \\
\hline & \multicolumn{4}{|c|}{ First season (2014) } & \multicolumn{4}{|c|}{ Second season (2015) } \\
\hline & \multicolumn{4}{|c|}{ Months after cutting planting } & \multicolumn{4}{|c|}{ Months after cutting planting } \\
\hline & 5 & 6 & 7 & Trea. av. & 5 & 6 & 7 & Trea. av. \\
\hline IBA at 4000 ppm & $88.87 \mathrm{ab}$ & 77.73abcd & 77.73abcd & $81.44 \mathrm{~A}$ & $88.87 \mathrm{ab}$ & $77.73 \mathrm{abc}$ & $100.00 \mathrm{a}$ & $88.87 \mathrm{~A}$ \\
\hline NAA at 500 ppm & 77.73abcd & 66.63bcde & $55.50 \mathrm{cdef}$ & $66.62 \mathrm{ABC}$ & $88.87 \mathrm{ab}$ & $66.60 \mathrm{bcd}$ & 44.40def & 66.62BCD \\
\hline $\begin{array}{l}\text { IBA at } 4000+\text { NAA at } 500 \\
\text { ppm }\end{array}$ & 77.73abcd & 55.50cdef & $66.63 \mathrm{bcde}$ & $66.61 \mathrm{ABC}$ & $77.73 \mathrm{abc}$ & $77.73 \mathrm{abc}$ & $100.00 \mathrm{a}$ & $85.16 \mathrm{~A}$ \\
\hline Moringa extr. at $10 \%$ & $11.10 \mathrm{~g}$ & $11.10 \mathrm{~g}$ & $11.10 \mathrm{~g}$ & $11.10 \mathrm{D}$ & 44.40def & $11.10 \mathrm{~g}$ & $11.10 \mathrm{~g}$ & $22.20 \mathrm{E}$ \\
\hline Mori & $11.10 \mathrm{~g}$ & $11.10 \mathrm{~g}$ & $11.10 \mathrm{~g}$ & $11.10 \mathrm{D}$ & 44.40def & $22.20 \mathrm{fg}$ & $22.20 \mathrm{fg}$ & $29.60 \mathrm{E}$ \\
\hline Garlic extr. at $10 \%$ & $100.00 \mathrm{a}$ & $66.63 \mathrm{bcde}$ & $66.63 \mathrm{bcde}$ & $77.73 \mathrm{AB}$ & $100.00 \mathrm{a}$ & $55.50 \mathrm{cde}$ & $66.60 \mathrm{bcd}$ & 74.03ABC \\
\hline Garlic extr. at $20 \%$ & $88.87 \mathrm{ab}$ & $66.63 \mathrm{bcde}$ & $66.63 \mathrm{bcde}$ & $74.03 \mathrm{AB}$ & $88.87 \mathrm{ab}$ & $66.60 \mathrm{bcd}$ & 77.73abc & $77.73 \mathrm{AB}$ \\
\hline Liquorice extr. at 5g/l & $88.87 \mathrm{ab}$ & $55.50 \mathrm{cdef}$ & 44.40 ef & $62.92 \mathrm{BC}$ & 77.73abc & 44.40def & $55.50 \mathrm{cde}$ & $59.21 \mathrm{CD}$ \\
\hline Liquorice extr.at10 g/l & $88.87 \mathrm{ab}$ & $55.50 \mathrm{cdef}$ & 77.73abcd & $74.03 \mathrm{AB}$ & 77.73abc & 77.73abc & 77.73abc & $77.73 \mathrm{AB}$ \\
\hline Yeast extr.at $5 \%$ & 44.40ef & $55.50 \mathrm{cdef}$ & 49.93def & $49.94 \mathrm{C}$ & 77.73abc & 44.40def & 33.30efg & $51.81 \mathrm{D}$ \\
\hline Yeast extr.at $10 \%$ & $66.63 \mathrm{bcde}$ & $49.93 \mathrm{def}$ & $33.30 \mathrm{fg}$ & $49.944 \mathrm{C}$ & $55.50 \mathrm{cde}$ & $49.93 \mathrm{cdef}$ & 44.40def & 49.94D \\
\hline Algae extr.at $2.5 \mathrm{~cm} / \mathrm{l}$ & 77.73abcd & 83.30abc & 77.73abcd & $79.59 \mathrm{AB}$ & 77.73abc & 77.73abc & 77.73abc & $77.73 \mathrm{AB}$ \\
\hline Algae at extr. $5 \mathrm{~cm} / \mathrm{l}$ & $83.30 \mathrm{abc}$ & $88.87 \mathrm{ab}$ & 77.73abcd & $83.30 \mathrm{~A}$ & 77.73abc & $77.73 \mathrm{abc}$ & 77.73abc & $77.73 \mathrm{AB}$ \\
\hline Date av. & $69.63 \mathrm{~A}$ & $57.22 \mathrm{~B}$ & $55.08 \mathrm{~B}$ & & $75.18 \mathrm{~A}$ & $57.65 \mathrm{~B}$ & $60.65 \mathrm{~B}$ & \\
\hline
\end{tabular}

Means in each column which have the same letter(s) are not significantly different.

Table 7. Effect of some natural extracts and synthetic growth regulators on total carbohydrates percentage in roots of Picual olive variety (2014 and 2015 seasons)

\begin{tabular}{|c|c|c|c|c|c|c|c|c|}
\hline \multirow[t]{4}{*}{ Treatment } & \multicolumn{8}{|c|}{ Total carbohydrates percentage of roots (\%) } \\
\hline & \multicolumn{4}{|c|}{ First season (2014) } & \multicolumn{4}{|c|}{ Second season (2015) } \\
\hline & \multicolumn{4}{|c|}{ Months after cutting planting } & \multicolumn{4}{|c|}{ Months after cutting planting } \\
\hline & 5 & $\overline{6}$ & 7 & Trea. av. & 5 & $\overline{6}$ & 7 & Av. trea. \\
\hline IBA at $4000 \mathrm{ppm}$ & $12.08 \mathrm{de}$ & $12.54 \mathrm{c}$ & $12.97 \mathrm{~b}$ & $12.53 \mathrm{~B}$ & $12.11 \mathrm{hi}$ & $12.53 \mathrm{f}$ & $13.09 \mathrm{c}$ & $12.57 \mathrm{~B}$ \\
\hline NAA at $500 \mathrm{ppm}$ & $11.55 \mathrm{ij}$ & 11.70hi & $11.97 \mathrm{ef}$ & $11.74 \mathrm{D}$ & $11.59 \mathrm{~m}$ & $11.90 \mathrm{j}$ & $12.30 \mathrm{~g}$ & $11.93 \mathrm{D}$ \\
\hline IBA at 4000 + NAA at $500 \mathrm{ppm}$ & $12.84 \mathrm{~b}$ & $12.86 \mathrm{~b}$ & $13.89 \mathrm{a}$ & $13.20 \mathrm{~A}$ & $12.91 \mathrm{~d}$ & $13.26 \mathrm{~b}$ & $13.87 \mathrm{a}$ & $13.34 \mathrm{~A}$ \\
\hline Moringa extr. at $10 \%$ & $9.65 \mathrm{w}$ & $9.85 \mathrm{uv}$ & $10.27 \mathrm{st}$ & $9.92 \mathrm{~L}$ & $10.17 \mathrm{z}$ & $10.27 y$ & $10.44 w$ & $10.29 \mathrm{M}$ \\
\hline Moringa extr. at $20 \%$ & $9.73 \mathrm{vw}$ & $9.93 \mathrm{u}$ & $10.45 \mathrm{qr}$ & $10.04 \mathrm{~K}$ & $10.45 \mathrm{w}$ & $10.51 \mathrm{uv}$ & $10.63 \mathrm{t}$ & $10.53 \mathrm{~K}$ \\
\hline Garlic extr. at $10 \%$ & $10.17 \mathrm{t}$ & $10.55 \mathrm{pq}$ & 10.860 & $10.53 \mathrm{H}$ & $10.65 \mathrm{t}$ & $11.19 \mathrm{p}$ & $10.98 \mathrm{r}$ & 10.94I \\
\hline Garlic extr. at $20 \%$ & $10.92 \mathrm{no}$ & $11.06 \mathrm{mn}$ & $11.33 \mathrm{kl}$ & $11.11 \mathrm{G}$ & $11.00 \mathrm{r}$ & $11.08 \mathrm{q}$ & $11.40 \mathrm{o}$ & $11.16 \mathrm{H}$ \\
\hline Liquorice extr. at 5g/l & 10.96 no & $11.21 \mathrm{~lm}$ & $11.65 \mathrm{hi}$ & $11.27 \mathrm{~F}$ & $11.03 \mathrm{qr}$ & $11.23 p$ & $11.591 \mathrm{~m}$ & $11.28 \mathrm{G}$ \\
\hline Liquorice extr.at10 g/l & $11.16 \mathrm{~m}$ & $11.33 \mathrm{kl}$ & $11.89 \mathrm{fg}$ & $11.46 \mathrm{E}$ & $11.20 \mathrm{p}$ & $11.53 \mathrm{n}$ & $11.85 \mathrm{jk}$ & $11.53 \mathrm{~F}$ \\
\hline Yeast extr.at $5 \%$ & $9.86 u v$ & $10.24 \mathrm{st}$ & $10.56 \mathrm{pq}$ & $10.22 \mathrm{~J}$ & $10.34 \mathrm{x}$ & $10.48 \mathrm{vw}$ & $10.64 \mathrm{t}$ & $10.49 \mathrm{~L}$ \\
\hline Yeast extr.at $10 \%$ & $9.94 \mathrm{u}$ & $10.35 \mathrm{rs}$ & $10.67 \mathrm{p}$ & $10.32 \mathrm{I}$ & $10.56 \mathrm{u}$ & $10.63 \mathrm{t}$ & $10.81 \mathrm{~s}$ & $10.67 \mathrm{~J}$ \\
\hline Algae extr.at $2.5 \mathrm{~cm} / \mathrm{l}$ & $11.43 \mathrm{jk}$ & $11.55 \mathrm{ij}$ & $12.18 \mathrm{~d}$ & $11.72 \mathrm{D}$ & $11.50 \mathrm{n}$ & 11.651 & $12.16 \mathrm{~h}$ & $11.77 \mathrm{E}$ \\
\hline Algae at extr. $5 \mathrm{~cm} / \mathrm{l}$ & $11.77 \mathrm{gh}$ & $11.95 \mathrm{ef}$ & $12.68 \mathrm{c}$ & $12.13 \mathrm{C}$ & $11.79 \mathrm{k}$ & $12.06 \mathrm{i}$ & $12.65 \mathrm{e}$ & $12.17 \mathrm{C}$ \\
\hline Date av. & $10.93 \mathrm{C}$ & $11.16 \mathrm{~B}$ & $11.64 \mathrm{~A}$ & & $11.18 \mathrm{C}$ & $11.41 \mathrm{~B}$ & $11.72 \mathrm{~A}$ & \\
\hline
\end{tabular}

Means in each column which have the same letter(s) are not significantly different. 
respectively, with significant differences with the other treatments. The higher levels of all natural extracts significantly exhibited higher carbohydrates content than the lower levels. Moringa extract at $10 \%$ treated cuttings contains significantly the lowest carbohydrates percentage (9.92 and $10.29 \%$ ) in both seasons, respectively, compared with the other treatments.

The total carbohydrates content in the root was gradually increased with advancing in planting months and reached the highest content seven months after planting (11.64 and 11.72\%) in the two seasons, respectively.

The all combinations of IBA at $4000 \mathrm{ppm}+$ NAA at $500 \mathrm{ppm} \times$ different dates gave the highest total carbohydrates content in roots with significant differences between them in both seasons.

The same trend in carbohydrates content in the shoots was observed in Table 8 in both seasons with the advance in planting months, and between treatments.

These results are in agreement with those reported by Ling and Zhong (2012) who elucidated that the highest total carbohydrates concentration and carbohydrate/nitrogen $(\mathrm{C} / \mathrm{N})$ ratio were recorded in the basal parts of the stem cuttings planted on 15 May, either before planting or 35 days after. A positive relationship of rooting percentage was found between total carbohydrates concentration and $\mathrm{C} / \mathrm{N}$ ratio. No consistent relationship was established between total nitrogen and rooting percentage. Results showed also high and low negative relationship of the rooting percentage of the cuttings between indole-3-acetic acid (IAA) concentration and gibberellins (GAs) concentration, respectively. In addition, 35 days after planting, a positive relationship was detected between abscisic acid (ABA) concentration and the rooting percentage. From these results, 15 May be recommended as the ideal planting date for improving the rooting and the vegetative growth of Tetraploid Locust stem cuttings compared to the other investigated planting dates. New shoot growth of Tetraploid Locust stopped in July, followed by the accumulation of carbohydrates because photosynthetic products were not exhausted by new shoot growth, and then the carbohydrates were converted into fructose and glucose from July to March of the next year.
Several studies indicated that auxins have the greatest effect on the initiation of adventitious roots and the division of root initials. Carbohydrates are particularly important as an energy source in the rooting process of cuttings (Fabbri et al., 2004). The reduction in the rooting percentage observed in the July cuttings may be attributed to that mother plants tended to flower at this time, causing the utilization of auxin for floral bud development and the depletion of the carbohydrates reserves (Hussein, 2003 and 2008).

Total carbohydrates and total nitrogen levels and $\mathrm{C} / \mathrm{N}$ ratio (Druege et al., 2004 ; Rapaka et al., 2005) have been reported to influence the adventitious rooting of plant species. Generally, nitrogen has been negatively correlated to rooting. Such effects have been suggusted to decreased carbohydrates levels and $\mathrm{C} / \mathrm{N}$ ratio, as important for root formation (Druege et al., 2000). In addition, Kasim et al. (2009) working on bitter almond found that high rooting ability was accompanied by their high $\mathrm{C} / \mathrm{N}$ ratio during the growth season.

Hanafy et al. (2012) cleared that the highest values of total carbohydrates were obtained with garlic extract followed by yeast extract, thus application of garlic extract resulted in the highest values of all recorded root characters, whereas the lowest values of all recorded characters were resulted by control treatment in both seasons.

\section{Total Soluble Phenols}

Table 9 declare that IBA at $4000 \mathrm{ppm}+$ NAA at $500 \mathrm{ppm}$ treatment achieved the highest total soluble phenols in the roots in both seasons compared with the other treatments. The higher concentrations of all natural extracts significantly gained the highest total soluble phenols than the lower concentrations for each natural extract.

The total soluble phenols in roots were decreased after six months then increased in the seventh month in both seasons.

The differences between combinations of algae at $2.5 \times 7$ months after planting and IBA at $4000 \mathrm{ppm}$ after 5 and 6 months were insignificant in the two seasons. The combinations of IBA at $4000 \mathrm{ppm}+\mathrm{NAA}$ at $500 \mathrm{ppm}$ after 7 months gave the highest significant total soluble phenols concentration in the roots compared with the other combinations in the two seasons. 
Table 8. Effect of some natural extracts and synthetic growth regulators on total carbohydrates percentage in shoots of Picual olive variety (2014 and 2015 seasons)

\begin{tabular}{lcccccccc}
\hline Treatment & \multicolumn{6}{c}{ Total carbohydrates percentage of shoots (\%) } \\
\cline { 2 - 9 } & \multicolumn{5}{c}{ First season (2014) } & \multicolumn{5}{c}{ Second season (2015) } \\
\cline { 2 - 9 } & \multicolumn{3}{c}{ Months after cutting planting } & \multicolumn{3}{c}{ Months after cutting planting } \\
\cline { 2 - 9 } & $\mathbf{5}$ & $\mathbf{6}$ & $\mathbf{7}$ & Trea. av. & $\mathbf{5}$ & $\mathbf{6}$ & $\mathbf{7}$ & Trea. av. \\
\hline IBA at 4000 ppm & $16.44 \mathrm{~d}$ & $16.75 \mathrm{~cd}$ & $17.15 \mathrm{c}$ & $16.78 \mathrm{~B}$ & $16.49 \mathrm{f}$ & $16.78 \mathrm{e}$ & $17.133 \mathrm{~d}$ & $16.80 \mathrm{~B}$ \\
NAA at 500 ppm & $14.49 \mathrm{fgh}$ & $15.82 \mathrm{e}$ & $16.40 \mathrm{~d}$ & $15.57 \mathrm{C}$ & $14.59 \mathrm{k}$ & $14.90 \mathrm{i}$ & $15.24 \mathrm{~h}$ & $14.91 \mathrm{C}$ \\
IBA at 4000 + NAA at 500 ppm & $18.41 \mathrm{~b}$ & $18.63 \mathrm{~b}$ & $19.19 \mathrm{a}$ & $18.74 \mathrm{~A}$ & $18.49 \mathrm{c}$ & $18.74 \mathrm{~b}$ & $19.16 \mathrm{a}$ & $18.80 \mathrm{~A}$ \\
Moringa extr. at 10\% & $11.26 \mathrm{u}$ & $11.83 \mathrm{rst}$ & $12.34 \mathrm{pq}$ & $11.81 \mathrm{I}$ & $11.54 \mathrm{e}$ & $12.27 \mathrm{a}$ & $12.37 \mathrm{z}$ & $12.06 \mathrm{M}$ \\
Moringa extr. at 20\% & $11.44 \mathrm{tu}$ & $11.95 \mathrm{qrs}$ & $12.55 \mathrm{op}$ & $11.98 \mathrm{HI}$ & $11.63 \mathrm{~d}$ & $12.45 \mathrm{y}$ & $12.54 \mathrm{x}$ & $12.21 \mathrm{~L}$ \\
Garlic extr. at 10\% & $13.45 \mathrm{n}$ & $15.66 \mathrm{e}$ & $14.09 \mathrm{~h}-\mathrm{k}$ & $14.40 \mathrm{E}$ & $13.49 \mathrm{u}$ & $13.74 \mathrm{~s}$ & $14.09 \mathrm{q}$ & $13.77 \mathrm{I}$ \\
Garlic extr. at 20 \% & $13.631 \mathrm{mn}$ & $13.93 \mathrm{j}-\mathrm{m}$ & $14.40 \mathrm{ghi}$ & $13.99 \mathrm{~F}$ & $13.71 \mathrm{~s}$ & $13.95 \mathrm{r}$ & $14.39 \mathrm{o}$ & $14.02 \mathrm{G}$ \\
Liquorice extr. at 5g/l & $13.53 \mathrm{mn}$ & $13.83 \mathrm{k}-\mathrm{n}$ & $14.14 \mathrm{~h}-\mathrm{k}$ & $13.83 \mathrm{~F}$ & $13.56 \mathrm{t}$ & $13.96 \mathrm{r}$ & $14.13 \mathrm{p}$ & $13.89 \mathrm{H}$ \\
Liquorice extr.at10 g/l & $13.81 \mathrm{k}-\mathrm{n}$ & $14.93 \mathrm{f}$ & $14.59 \mathrm{fg}$ & $14.45 \mathrm{E}$ & $13.94 \mathrm{r}$ & $14.08 \mathrm{q}$ & $14.49 \mathrm{~m}$ & $14.17 \mathrm{~F}$ \\
Yeast extr.at 5 \% & $11.54 \mathrm{stu}$ & $12.23 \mathrm{pqr}$ & $12.64 \mathrm{op}$ & $12.14 \mathrm{H}$ & $11.78 \mathrm{c}$ & $12.44 \mathrm{y}$ & $12.65 \mathrm{w}$ & $12.29 \mathrm{~K}$ \\
Yeast extr.at 10 \% & $12.34 \mathrm{pq}$ & $12.47 \mathrm{op}$ & $12.84 \mathrm{o}$ & $12.55 \mathrm{G}$ & $11.98 \mathrm{~b}$ & $12.54 \mathrm{x}$ & $12.83 \mathrm{v}$ & $12.45 \mathrm{~J}$ \\
Algae extr.at $\mathbf{2 . 5} \mathbf{~ c m / l ~}$ & $14.03 \mathrm{i}-1$ & $13.99 \mathrm{i}-1$ & $14.93 \mathrm{f}$ & $14.32 \mathrm{E}$ & 14.551 & $14.46 \mathrm{mn}$ & $14.91 \mathrm{i}$ & $14.64 \mathrm{E}$ \\
Algae at extr. 5 cm/l & $14.34 \mathrm{~g}-\mathrm{j}$ & $14.63 \mathrm{fg}$ & $15.39 \mathrm{e}$ & $14.79 \mathrm{D}$ & $14.45 \mathrm{n}$ & $14.86 \mathrm{j}$ & $15.35 \mathrm{~g}$ & $14.89 \mathrm{D}$ \\
Date av. & $13.75 \mathrm{C}$ & $14.36 \mathrm{~B}$ & $14.67 \mathrm{~A}$ & & $13.86 \mathrm{C}$ & $14.24 \mathrm{~B}$ & $14.56 \mathrm{~A}$ & \\
\hline
\end{tabular}

Means in each column which have the same letter(s) are not significantly different.

Table 9. Effect of some natural extracts and synthetic growth regulators on total soluble phenols in roots of Picual olive variety (2014 and 2015 seasons)

\begin{tabular}{|c|c|c|c|c|c|c|c|}
\hline \multirow[t]{4}{*}{$\overline{\text { Treatment }}$} & \multicolumn{7}{|c|}{ Total soluble phenols of roots $(\mathrm{mg} / \mathrm{g}$. DW) } \\
\hline & \multicolumn{4}{|c|}{ First season (2014) } & \multicolumn{3}{|c|}{ Second season (2015) } \\
\hline & \multicolumn{4}{|c|}{ months after cutting planting } & \multicolumn{3}{|c|}{ Months after cutting planting } \\
\hline & 5 & 6 & 7 & Trea. av. & 5 & 7 & Trea. av. \\
\hline IBA at 4000 ppm & $317.50 \mathrm{ef}$ & $320.37 \mathrm{e}$ & $373.07 \mathrm{c}$ & $336.98 \mathrm{C}$ & 332.73 & 335.40 f $370.70 d$ & $346.28 \mathrm{~B}$ \\
\hline NAA at 500 ppm & $390.63 \mathrm{~b}$ & $305.27 \mathrm{fg}$ & $342.13 \mathrm{~d}$ & $346.01 \mathrm{~B}$ & 272.00 & $277.27 \mathrm{~m} \mathrm{311.20j}$ & $286.82 \mathrm{E}$ \\
\hline IBA at 4000 + NAA at 500 ppm & $374.73 \mathrm{c}$ & $383.63 \mathrm{bc}$ & $431.07 \mathrm{a}$ & $396.48 \mathrm{~A}$ & 391.53 & $402.00 \mathrm{~b} 430.83 \mathrm{a}$ & $408.12 \mathrm{~A}$ \\
\hline Moringa extr. at $10 \%$ & 195.33 o-r & $186.33 \mathrm{r}$ & $217.47 \mathrm{mn}$ & $199.71 \mathrm{~J}$ & 214.93 & 213.70 A $232.93 \mathrm{t}$ & $220.52 \mathrm{~K}$ \\
\hline Moringa extr. at $20 \%$ & $204.80 \mathrm{n}-\mathrm{q}$ & $191.53 \mathrm{qr}$ & $231.77 \mathrm{kl}$ & 209.37GHI & I 237.43 & $219.07 \times 237.90 \mathrm{r}$ & $47 \mathrm{G}$ \\
\hline Garlic extr. at $10 \%$ & 197.60 o-r & $189.90 \mathrm{r}$ & $228.43 \mathrm{~lm}$ & 205.31 HIJ & 210.9 & 201.63 D 228.07 & $213.54 \mathrm{M}$ \\
\hline Garlic extr. at $20 \%$ & 206.40 no & $191.90 \mathrm{qr}$ & $234.50 \mathrm{kl}$ & $210.93 \mathrm{GH}$ & I 232.20 & 216.43 y $234.50 \mathrm{~s}$ & $227.71 \mathrm{I}$ \\
\hline Liquorice extr. at $5 \mathrm{~g} / \mathrm{l}$ & $203.60 \mathrm{opq}$ & $162.80 \mathrm{~s}$ & $242.83 \mathrm{jk}$ & $203.08 \mathrm{IJ}$ & 226.00 & v 213.33 A $242.13 q$ & $227.16 \mathrm{~J}$ \\
\hline Liquorice extr.at10 g/l & $221.90 \mathrm{~lm}$ & $244.90 \mathrm{jk}$ & $295.73 \mathrm{gh}$ & $254.18 \mathrm{~F}$ & 233.83 & 276.07 n $294.13 \mathrm{k}$ & $268.01 \mathrm{~F}$ \\
\hline Yeast extr.at $5 \%$ & $191.43 \mathrm{qr}$ & 194.00 o-r & $226.47 \mathrm{~lm}$ & 203.97HIJ & 211.10 & 212.60 B $225.60 \mathrm{w}$ & $216.43 \mathrm{~L}$ \\
\hline Yeast extr.at $10 \%$ & 205.70 nop & $192.53 \mathrm{pqr}$ & $249.50 \mathrm{j}$ & $215.91 \mathrm{G}$ & 231.53 & 215.37 z $237.90 \mathrm{r}$ & $228.27 \mathrm{H}$ \\
\hline Algae extr.at $2.5 \mathrm{~cm} / \mathrm{l}$ & $265.63 \mathrm{i}$ & $256.13 \mathrm{ij}$ & $311.93 \mathrm{ef}$ & $277.90 \mathrm{E}$ & 283.4 & 273.00 о 312.20 & $289.54 \mathrm{D}$ \\
\hline Algae at extr. $5 \mathrm{~cm} / \mathrm{l}$ & $290.50 \mathrm{~h}$ & $297.00 \mathrm{gh}$ & $343.60 \mathrm{~d}$ & 310.37 D & 312.90 & $318.00 \mathrm{~h} 342.00 \mathrm{e}$ & $324.30 \mathrm{C}$ \\
\hline Date av. & $251.21 \mathrm{~B}$ & $239.72 \mathrm{C}$ & $286.81 \mathrm{~A}$ & & $260.81 \mathrm{H}$ & 3259.53 C 284.62 A & \\
\hline
\end{tabular}

Means in each column which have the same letter(s) are not significantly different. 
Total soluble phenols in shoots were gradually increased as presented in Table 10 . The IBA at $4000 \mathrm{ppm}+\mathrm{NAA}$ at $500 \mathrm{ppm}$ treatment achieved the highest values (532.31 and $520.19 \mathrm{mg} / \mathrm{g} \mathrm{DW}$ ) in the first and second seasons, respectively, while moringa extract at $10 \%$ was the lowest value in the first season (329.43 $\mathrm{mg} / \mathrm{g}$. DW) and yeast at $10 \%$ in the second season (346.97 $\mathrm{mg} / \mathrm{g} \mathrm{DW})$. The other treatments were inbetween.

Aslmoshtaghi and Shahsavar (2010b) reported that there were significant differences between the tested cultivars in the total phenolic content and the highest phenolic compounds were found in "Konsrvalia" after 120 days and the lowest phenolic compounds were found in "Roghani" cultivar. In addition, Denaxa et al. (2012) showed that the pattern of changes in sugar concentrations at 3 and 7 days after planting was different for each cultivar. Initial internal sugar concentrations and their metabolism might be important during the early period of the rooting process.

Krajnc et al. (2012) cleared that, the twenty three days after severance a transient decline in total protein contents was accompanied by an increased accumulation of total phenolics in all Kelpak (brown algae) applications. Thirty six days after severance, a more than $220 \%$ increase in total protein contents and a 50\% decline in total phenolics were recorded in untreated cuttings when compared to the previous sampling date.

Abdalla (2013) showed that Moringa oleifera extracts increased the amounts of each of total sugars and phenols in rocket plants.

Taha et al. (2016) showed that application of yeast extract at 10, 15 and $20 \%$ resulted in the highest contents of total soluble phenols and total soluble sugars.

Table 10. Effect of some natural extracts and synthetic growth regulators on total soluble phenols in shoots of Picual olive variety (2014 and 2015 seasons)

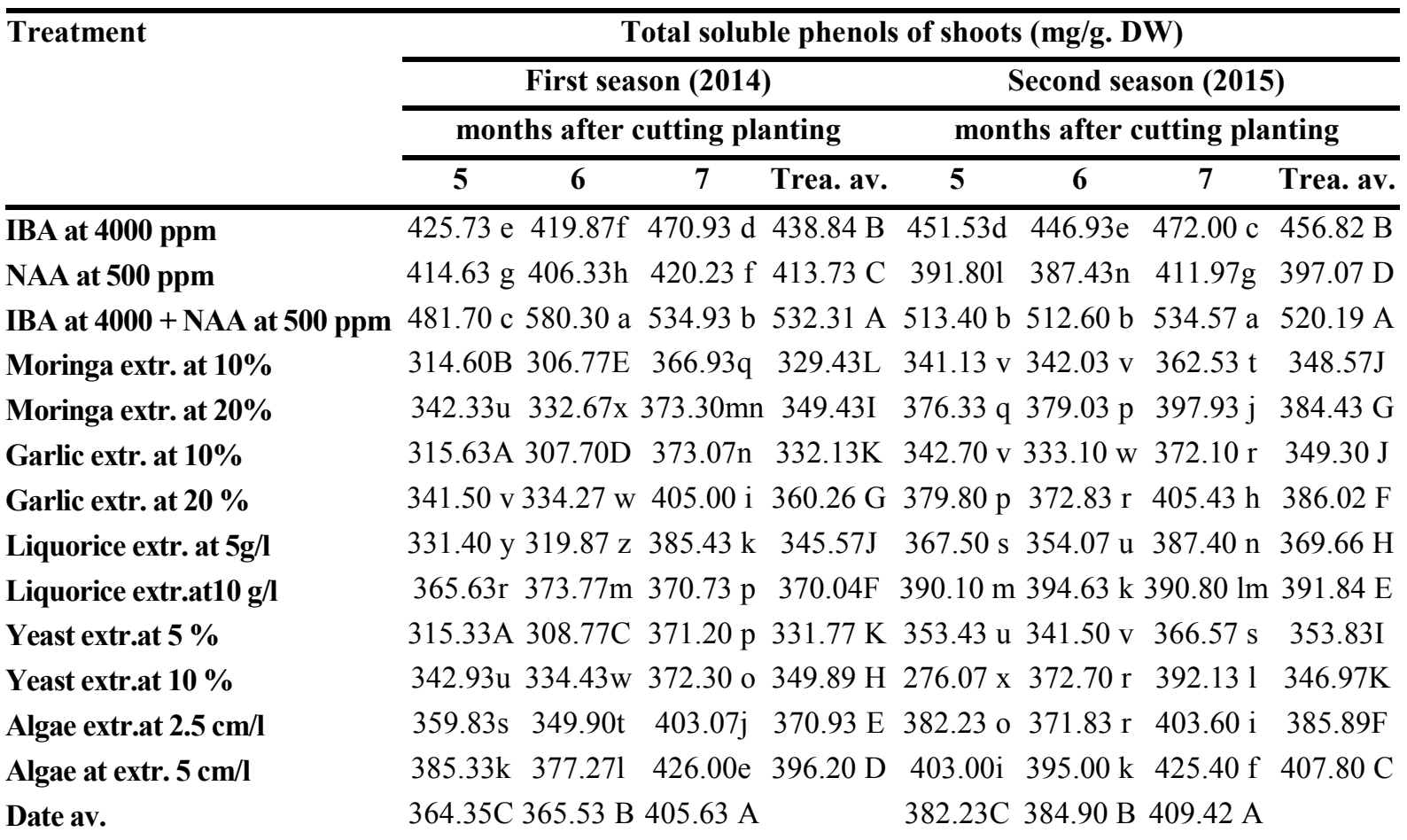

Means in each column which have the same letter(s) are not significantly different. 


\section{REFERENCES}

Abd El-Moniem E.A. and A.S.E. Abd-Allah (2008). Effect of green algae cells extract as foliar spray on vegetative growth, yield and berries quality of Superior grapevines. Ame.Eurasian J. Agric. and Environ. Sci., 4 (4): 427-433.

Abd El-Migeed, A.A., A.B. El-Sayed and H.S.A. Hassan (2004). Growth enhancement of olive transplants by broken cells of fresh green algae as soil application. Minufia J. Agric. Res., 29: 723-737.

Abdalla, M.M. (2013). The potential of Moringa oleifera extract as a biostimulant in enhancing the growth, biochemical and hormonal contents in rocket (Eruca vesicaria subsp. sativa) plants. Int. J. Plant Physiol. and Biochem., 5 (3): 42-49.

Abdulrahman, A.S. (2013). Effect of foliar spray of ascorbic acid, zinc, seaweed extracts and biofertilizer (EM1) on growth of almonds (Prunus amygdalus) Seedling. Int. J. Pure Appl. Sci. Technol., 17(2) : 62-71.

Agele, S.O, O.P. Aiyelari and E.A. Obi (2013). Pattern of rooting and growth of cuttings of some species of insecticidal and medicinal importance as affected by growth promoting substances. Octa J. Env. Res., 1 (2): 151-160.

Andrade, R. A. D., and A. B. G. Martins (2003). Vegetative propagation for citrus rootstocks. Revista Brasileira de Fruticultura, 25 (1): 134-136.

AOAC (2012). Official Methods of Analysis, International, $19^{\text {th }} \mathrm{Ed}$. Association of Official Analytical Chemists, Gaithersburg, Maryland, USA.

Arthur, G.D., W.A. Stirk and J. Van staden (2003). Effect of a seaweed concentrate on the growth and yield of three varieties of Capsicum annuum. S. Afr. J. Bot., 69 : 207211.

Aslmoshtaghi, E. and A.R. Shahsavar (2010a). Endogenous promoters and inhibitors in olive cuttings in relation to adventitious root formation. J. Biol. Environ. Sci., 4 (12): 143148 .
Aslmoshtaghi, E. and A.R. Shahsavar (2010b). Endogenous soluble sugars, starch contents and phenolic compounds in easy- and difficult-to-root olive cuttings. J. Biol. Environ. Sci., 49 (11): 83-86.

Aslmoshtaghi, E., A.R. Shahsavar and M.R. Taslimpour (2014). Effects of IBA and Putrescine on root formation of olive cuttings. Agric. Conspectus Scientifi cus., 79 (3): 191-194 .

Ayoub, S.J. (1995). Improving rooting of semihardwood 'Nabali' olive cuttings. M.Sc. Thesis, Jordan Univ., Amman, Jordan.

Babilie, R., M. Jbour and B. Abu Trabi (2015). Effect of foliar spraying with licorice root and seaweed extracts on growth and seed production of onion (Allium cepa L.). Int. J. Chem. Tech. Res., 8 (11): 557-563.

Cheng and Hanning (1955). Phenolic compound in potato Tis-sue. Food Res., $20: 506-511$.

Da Silva, J.A.T., J. Dobránszki and S. Ross (2013). Phloroglucinol in plant tissue culture. In vitro Cellular and Develop. Biol. Plant, 49 (1): 1-16.

Denaxa, N.K., S.N. Vemmos and P.A. Roussos (2012). The role of endogenous carbohydrates and seasonal variation in rooting ability of cuttings of an easy and a hard to root olive cultivars (Olea europaea L.). Scientia Hort., $143: 19-28$.

Dolor, D.E., N. Ehondor and L. Odiete (2010). Effect of some seed extracts and IBA on the rooting of leafy stem cuttings of Irvingiawombolu (Vermoesen). Agric. Tropica et Subtropica, 43(1): 11-18.

Druege, U., S. Zerche, R. Kandner and M. Ernst (2000). Relation between nitrogen status, carbohydrate distribution and subsequent rooting of Chrysanthemum cuttings as affected by pre-harvest nitrogen supply and cold-storage. Ann. Bot., 85: 687- 701.

Druege, U., S. Zerche and R. Kandner (2004). Nitrogen and storage-affected carbohydrate partitioning in high-light-adapted pelargonium cuttings in relation to survival and adventitious root formation under low light. Ann. Bot., 94: 831-832. 
Duncan, D.B. (1958). Multiple Rang and Multiple F test. Biomet., 11: 1-42.

El-Ghayaty, S.H., M.A. El-Serafy, E.S. Hegazy and A.E. Abd El-Monim (2010). Studies on the rooting of olive cuttings. J. Plant Prod., 1 (1): $1-10$.

El-Shayeb, N.S.A. (2009). Physiological studies. Oenotherabiennis (Bio-fertilizer and plant extracts) Ph.D. Thesis, Hort. Dept. Fac. Agric., Benha Univ., Egypt.

Fabbri, A., G. Bartolini, M. Lombardi and S. Kailis (2004). Olive propagation manual. CSIRO Publishing, Melbourne, Aust.

Fernandes, J.M., M.C. Serrano and E. Amaral (2002). Effect of different hormone treatments on rooting of Olea europaea cv. Galega vulgar cuttings, Acta Hort., 586 : 875-877.

Foidl, N., H.P.S. Makkar and K. Becker (2001). The potential of Moringa oleifera for agricultural and industrial uses. In: L.J. Fuglie (Ed.), The Miracle Tree: The Multiple Attributes of Moringa, 45-76.

Fuglie, L.J. (2000). The Miracle Tree: Moringa oleifera: Natural Nutrition for the Tropics. The multiple Attributes of Moringa, 172.

Gill, J.K., H. Singh, A. Thakur and S.K. Jawandha (2014). Studies on simultaneous grafting and rooting of peach on Flordaguard rootstock. Hort. Flora Res. Spectrum, 3 (3): 259-262.

Gollan J.R. and J.T. Wright. (2006). Limited grazing pressure by native herbivores on the invasive seaweed caulerpa, Taxi folia in a temperate, Aust. Estuary Marine and Freshwater Res., 57 (7):685-694.

Hamooh, B.T. (2014). The Effects of cutting collection time, auxin types and auxin concentrations on rooting of olive (Olea europaea L.) cuttings under arid land conditions. Int. J. Eng. Res. and Technol. (IJERT), 3 (1): 1389 - 1395.

Hanafy, M.S., F.M. Saadawy, S.M.N. Milad and R.M. Ali (2012). Effect of some natural extracts on growth and chemical constituents of Schefflera arboricola Plants. J. Hort. Sci. and Ornamental Plants, 4 (1): 26-33.
Hartmann, H.T., D.E. Kester, F.T. Davies and R.L. Geneve (2002). Plant Propagation, Principles and Practices. $7^{\text {th }}$ Ed., Prentice Hall, New Jersey, 880.

Hussein, M.M.M. (2003). Effect of planting dates and indole-butyric acid on rooting of Beaumontia grandiflora, Wallich cuttings and consequent plant growth . Arab Univ J Agric Sci, Ain Shams Univ, Cairo, 11 : 765787.

Hussein, M.M.M. (2008). Studies on the rooting and the consequent plant growth on the stem cuttings of Thunbergia grandiflora, (Roxb ex Rott1.) Roxb1- effect of different planting dates .World J. Agric. Sci., 4: 125-132.

Ibrahim, Z.R. (2013). Effect of foliar spray of ascorbic acid, Zn, seaweed extracts (Sea Force) and Biofertilizers (EM-1) on vegetative growth and root growth of olive (Olea Europaea L.) transplants cv. Hojblanca. Int. J. Pure Appl. Sci. Technol., 17 (2): 79-89.

Ismaili, H. and H. Fiku (2010). Olive cultivar propagation based on Nebulization method. Albanian J. Agric. Scientia, 10: 2218-2020.

Jan, I., M. Sajid, A. Rab, A. Iqbal, O. Khan, Y. Jamal and S.T. Shah (2014). Effect of various concentrations of Indole butyric acid (IBA) on olive cuttings. Mitteilungen Klosterneuburg, 64: 127-136.

Jana, B.R., B. Das and S. Kumar (2015). Efficacy of IBA and determination of cuttings size in Asian pear (Pyrus pyrifolia L.). Int. J. Plant Res., 5(3): 64-67.

Jiang, W.J., D.Y. Qu, D. Mu and L.R. Wang (2004). Protected cultivation of horticultural crops in China. Hort. Rev., 30: 115-162.

Jones, N.S. and J. van Staden (1997). The effect of a seaweed application on the rooting of pine cuttings. S. Afr. J. Bot., 6 (3): 141- 145.

Kasim, N.E., M.S. Abou Rayya, M.A. Shaheen, T.A. Yehia and E.L. Ali (2009). Effect of different collection times and some treatments on rooting and chemical internal constituents of Bitter Almond hardwood cuttings. Res. J. Agric. and Biol. Sci., 5: 116122. 
Kelen, M. and G. Ozkan (2003). Relationships between rooting ability and changes of endogenous IAA and ABA during the rooting of hardwood cuttings of some grapevine rootstocks. Europ. J. Hort. Sci., 68: 8-13.

Khajehpour, G., V. Jameizadeh and N. Khajehpour (2014). Effect of different concentrations of IBA (Indole butyric acid) hormone and cutting season on the rooting of the cuttings of olive (Olea europaea Var Manzanilla) . Int. J. Adv. Biol. Biom. Res., 2 (12): 2920-2924.

Krajnc, A.U., A. Ivanuš, J. Kristl and A. Šušek (2012). Seaweed extract elicits the metabolic responses in leaves and enhances growth of Pelargonium cuttings. Europ. J. Hort. Sci., 77 (4): 170-181.

Lazaj, A., P. Rama and H. Vrapi (2015). The interaction with season collection of cuttings, Indol butyric acid (IBA) and juvenility factors on root induction in Olea europaea L. (Cultivar "Kalinjot"). Int. Ref. J. Eng. and Sci. (IRJES), 4 (3): 32-38.

Ling, W.X. and Z. Zhong (2012). Seasonal variation in rooting of the cuttings from Tetraploid Locust in relation to nutrients and endogenous plant hormones of the shoot. Turk J. Agric., 36: 257-266.

Mansour, A.E., Gh. Cimpoies and F.F. Ahmed (2006). Application of algae extract and boric acid for obtaining higher yield and better fruit quality of Anna apple, Stiinta Agricola, 2, 14-20.

Martins, A.B.G., F.A. Graciano and A.V.C.D. Silva (2001). Cloning rose apple (Syzygium malacensis) by cutting of leaves branches. Revista Brasileira de Fruticultura, 23 (2): 365-368.

Mayer, N.A., G.L. Reighard and W. Bridges (2013). Peach rootstock propagation under intermittent mist system. In VIII Int. Peach Symposium, 1084: 53-62.

Mayer, N.A., L. Picolotto, P.V. Bastos, B. Ueno and L.E.C. Antunes (2014). Herbaceous cutting propagation of peach rootstocks in late summer. Semina: Ciências Agrárias, 35 (4): 1761-1772.
Mohamed, Y.I. (2015). Evaluation the effect of rooting media and hormonal concentrations (IBA) in three olive cultivars growing in Siwa Oesis-Egypt. Int. J. Agric. Sci., 5 (3): 500-503.

Moore, L.W., G. Warren and G. Strobel (1979). Involvement of a plasmid in the hairy root disease of plants caused by Agrobacterium rhizogenes. Plasmid., 2 : 617-626.

Nagodawithana, W.T. (1991). Yeast technology. Foods Corporation Pup. By Van Nostrand Reinhold New York, USA, 273.

Negash, L. (2003). Vegetative propagation of the threatened African wild olive [Olea europaea L. sub sp. cuspidata (Wall. ex DC.) Ciffieri]. New Forests, 26 (2): 137-146.

Németh, Z.S. (2011). Effect of different growth stimulants for rooting of prunus cuttings. OTDK, 34.

Osterc, G. and F. Štampar (2008). Initial cutting lenght modifies polyphenol profile in Castanea cuttings during the root formation process. Eur. J. Hort. Sci., 73: 201-204.

Patil, V.N., P.S. Chauhan, R.S. Shivankar, S.H. Vilhekar and V.S. Waghmare (2001). Effect of plant growth regulators on survival and vegetative growth of grapevine cuttings. Agric. Sci. Digest, 21 (2): 97-99.

Peixe, A., A. Raposo, R. Lourenco, H. Cardoso, and E. Macedo (2007). Coconut water and BAP successfully replaced zeatin in olive (Olea europaea L.) micropropagation. Scienia Hort., 113:1-7.

Perilli, S., L. Moubayidin and S. Sabatini (2010). The molecular basis of cytokinin function. Curr. Opin. Plant Biol., 13: 21-26.

Pio, R., D. Costabastos and A.J. Berti (2005). Rooting of different types of olive tree cutting using indol butyric acid. Cienc Agrotec Lavras, 29: 562-567.

Pop, T.I., D. Pamfil and C. Bellini (2011). Auxin Control in the Formation of Adventitious Roots. Not. Bot. Hort. Agrobot. Cluj., 39: 307-316.

Porghorban, M., E. G. Moghadam and A. Asgharzadeh (2014). Effect of media and indole butyric acid (IBA) concentrations on 
rooting of Russian olive (Elaeagnus angustifolia 1.) Semi-hard wood cuttings. Indian J. Fundamental and Appl. Life Sci., 4 (3) : 517-522.

Quaddoury, A. and M. Amssa (2004). Effect of exogenous indole butyric acid on root formation and peroxidase and indole-3-acetic acid oxidase activities and phenolic contents in date palm offshoots. Bot. Bull. Acad. Sinica, 45 : 127-131.

Rapaka, V.K., B. Bessler, M. Schreiner and U. Druege (2005). Interplay between initial carbohydrate availability, current photosynthesis, and adventitious root formation in Pelargonium cuttings. Plant Sci., 168: 15471560 .

Sachan, D., S.K. Jain and N. Singh (2011). In vitro and in vivo efficacy of Moringa oleifera plant constituents in urolithiasis as antilithiatic drug. Int. J. Pharmac. Sci. Res. (IJPSR) 2 (7): 1638-1644.

Sadak, M.S. (2016). Physiological role of yeast extract and nicotinamide on Pisum sativum L. plants under heat stress. Int. J. Pharm. Tech. Res., 9 (9): 170-178.

Samaan, L.G., E.F.A. El-Dengawy and M.E. ElZayat (2010). Response of hardwood stems cuttings of guava (Psidium guajava L.) to various growth and root promoting regulators. J. Plant Prod., 1 (2): 301 - 317.

Sebastiani, L. and R. Tognetti (2004). Growing season and hydrogen peroxide effects on root induction and development in Olea europaea L.(cvs 'Frantoio'and 'Gentile di Larino') cuttings. Scientia Hort., 100 (1): 75-82.

Shabani, L., A.A. Ehsanpour, G. Asghari and J. Emami (2009). Glycyrrhizin production by in vitro cultured Glycyrrhiza glabra elicited by Methyl Jasmonate and salicylic acid. Russian J. Plant Physiol., 56: 621-626.

Sharma, Y., D.D. Sharma and K. Singh (2014). Studies on the propagation of apple clonal rootstock Merton 793 through hardwood cuttings. Asian J. Hort., 9 (1): 128-131.
Snedecor, G.W. and W.G. Cochran (1990). Statistical Methods $7^{\text {th }}$ Ed. The lowa state. Univ. press. Arnes. Iowa. USA, 593.

Soni, N., S.K. Pandey, S.S. Singh, S.R.K. Singh, A. Mishra, S.S. Baghel and P.K. Kaurav (2016). Propagation of guava through cuttage under net house condition at Jabalpur, Madhya Pradesh, India. Flora and Fauna, 22 (1): 36-40.

Spinelli, F., F. Giovanni, N. Massimo, S. Mattia and C. Guglielmo (2009). Perspectives on the use of a seaweed extract to moderate the negative effects of alternate bearing in apple trees, J. Hort. Sci. Biotechn., 17(1): 131-137.

Taha, L.S., S.M.M. Ibrahim and N.G. Abdel Aziz (2016). Vegetative growth, chemical composition, and flavonoids content of Azadirachta indica plants as affected by application of yeast natural extract. J. Appl. Pharmaceutical Sci., 6 (04): 093-097.

Thorsen, M.K., S. Woodward and B.M. McKenzie (2010). Kelp (Laminaria digitata) increases germination and affects rooting and plant vigour in crops and native plants from anarable grassland in the Outer Hebrides, Scotland. J. Coast Conserv, 14:239 -247.

Urbanek, K.A., A. Ivanuš, J. Kristl and A. Šušek (2012). Seaweed extract elicits the metabolic responses in leaves and enhances growth of pelargonium cuttings. Europ. J. Hort. Sci., 77 (4): 170-181.

Vinoth, S., P. Gurusaravanan and N. Jayabalan (2012). Effect of seaweed extracts and plant growth regulators on high-frequency in vitro mass propagation of Lycopersicon esculentum L. (tomato) through double cotyledonary nodal explants. J. Appl. Phycol., 24: 13291337.

Wiesman, Z. and S. Lavee (1994). Vegetative growth retardation, improved rooting and viability of olive cuttings in response to application of growth retardants. Plant Growth Regul., 14: 83-90.

Zaki, S.S. and M.M. Rady (2015). Moringa oleifera leaf extract improves growth, physiochemical attributes, antioxidant defence system and yields of salt-stressed Phaseolus vulgaris L. plants. Int. J. Chem. Tech. Res., 8 (11): 120-134. 


\title{
تأثير بعض المستخلصات الطبيعية وإندول حمض البيوتريك ونفتالين حمض الخليك على تجذير عقل الزيتون صنف بيكوال
}

\author{
عبدالله محمد حسن عيد ـ صفاء عبدالغني نمير - محمد محمود إبراهيم - محمد ممتاز جاد \\ قسم البساتين ـ كلبة الزر اعة - جامعة الزقازيق ـ مصر
}

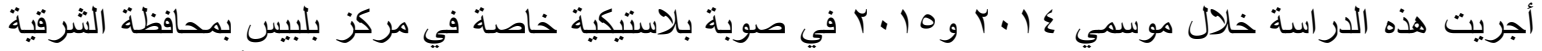

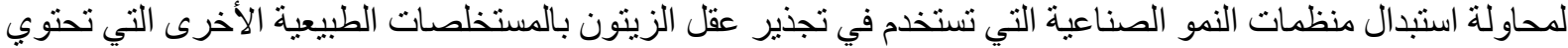

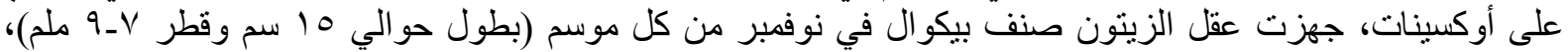

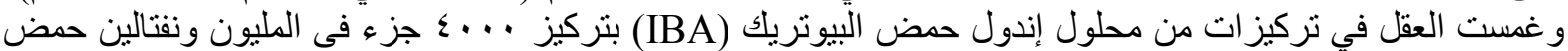

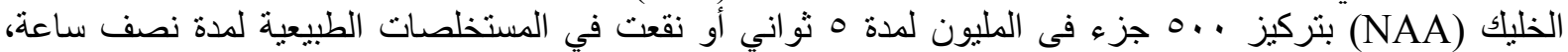

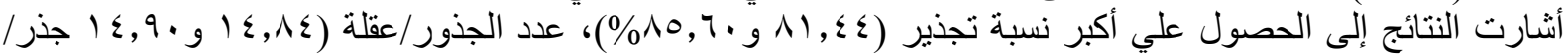

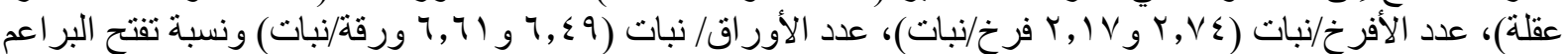

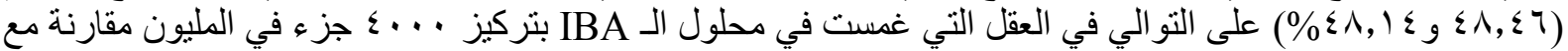

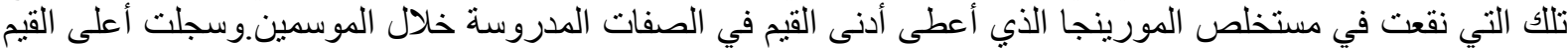

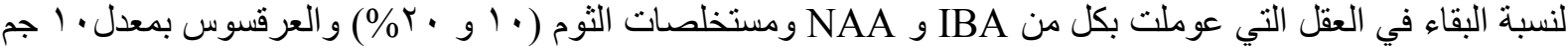

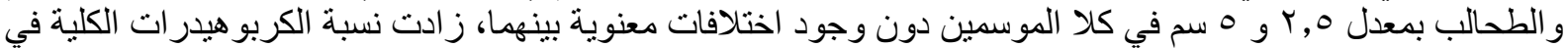

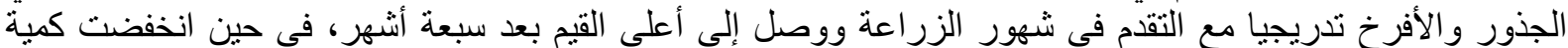

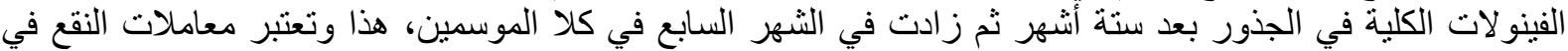

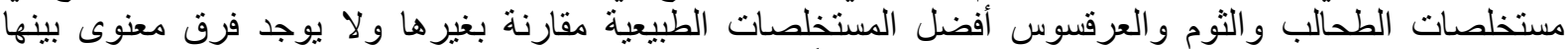

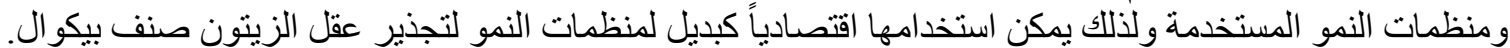

أستاذ البساتين - كلية الزر اعة بمشتهر - جامعة بنها. أستاذ الفاكهة المتفرغ النين - كلية الزر اعة - جامعة الزقازيق. 